\title{
Finite element modeling effects of inter-yarn friction on the single-layer high-performance fabrics subject to ballistic impact
}

DOI:

10.1016/j.mechmat.2018.08.003

\section{Document Version}

Accepted author manuscript

Link to publication record in Manchester Research Explorer

Citation for published version (APA):

Chu, Y., \& Chen, X. (2018). Finite element modeling effects of inter-yarn friction on the single-layer highperformance fabrics subject to ballistic impact. Mechanics of Materials, 126, 99-110.

https://doi.org/10.1016/j.mechmat.2018.08.003

\section{Published in:}

Mechanics of Materials

\section{Citing this paper}

Please note that where the full-text provided on Manchester Research Explorer is the Author Accepted Manuscript or Proof version this may differ from the final Published version. If citing, it is advised that you check and use the publisher's definitive version.

\section{General rights}

Copyright and moral rights for the publications made accessible in the Research Explorer are retained by the authors and/or other copyright owners and it is a condition of accessing publications that users recognise and abide by the legal requirements associated with these rights.

\section{Takedown policy}

If you believe that this document breaches copyright please refer to the University of Manchester's Takedown Procedures [http://man.ac.uk/04Y6Bo] or contact uml.scholarlycommunications@manchester.ac.uk providing relevant details, so we can investigate your claim.

\section{OPEN ACCESS}




\section{Accepted Manuscript}

Finite element modeling effects of inter-yarn friction on the single-layer high-performance fabrics subject to ballistic impact

Yanyan Chu, Xiaogang Chen

PII: S0167-6636(18)30247-3

DOI: https://doi.org/10.1016/j.mechmat.2018.08.003

Reference: MECMAT 2911

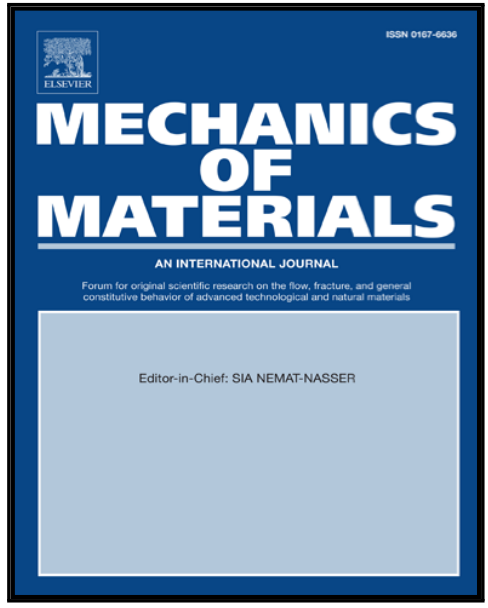

To appear in:

Mechanics of Materials

Received date: $\quad 7$ April 2018

Revised date: $\quad 6$ August 2018

Accepted date: $\quad 7$ August 2018

Please cite this article as: Yanyan Chu , Xiaogang Chen , Finite element modeling effects of inter-yarn friction on the single-layer high-performance fabrics subject to ballistic impact, Mechanics of Materials (2018), doi: https://doi.org/10.1016/j.mechmat.2018.08.003

This is a PDF file of an unedited manuscript that has been accepted for publication. As a service to our customers we are providing this early version of the manuscript. The manuscript will undergo copyediting, typesetting, and review of the resulting proof before it is published in its final form. Please note that during the production process errors may be discovered which could affect the content, and all legal disclaimers that apply to the journal pertain. 
Highlights

- Higher inter-yarn friction will make the energy absorption rate increase monotonously.

- Higher inter-yarn friction will make the response mode of fabric more globalized.

- Near zero friction, strain energy(SE) is the dominant mechanism of a fabric while at higher

- inter-yarn friction, kinetic energy(KE) becomes the dominant one

- An maximum inter-yarn friction exists for frictional dissipation energy(FDE) absorption

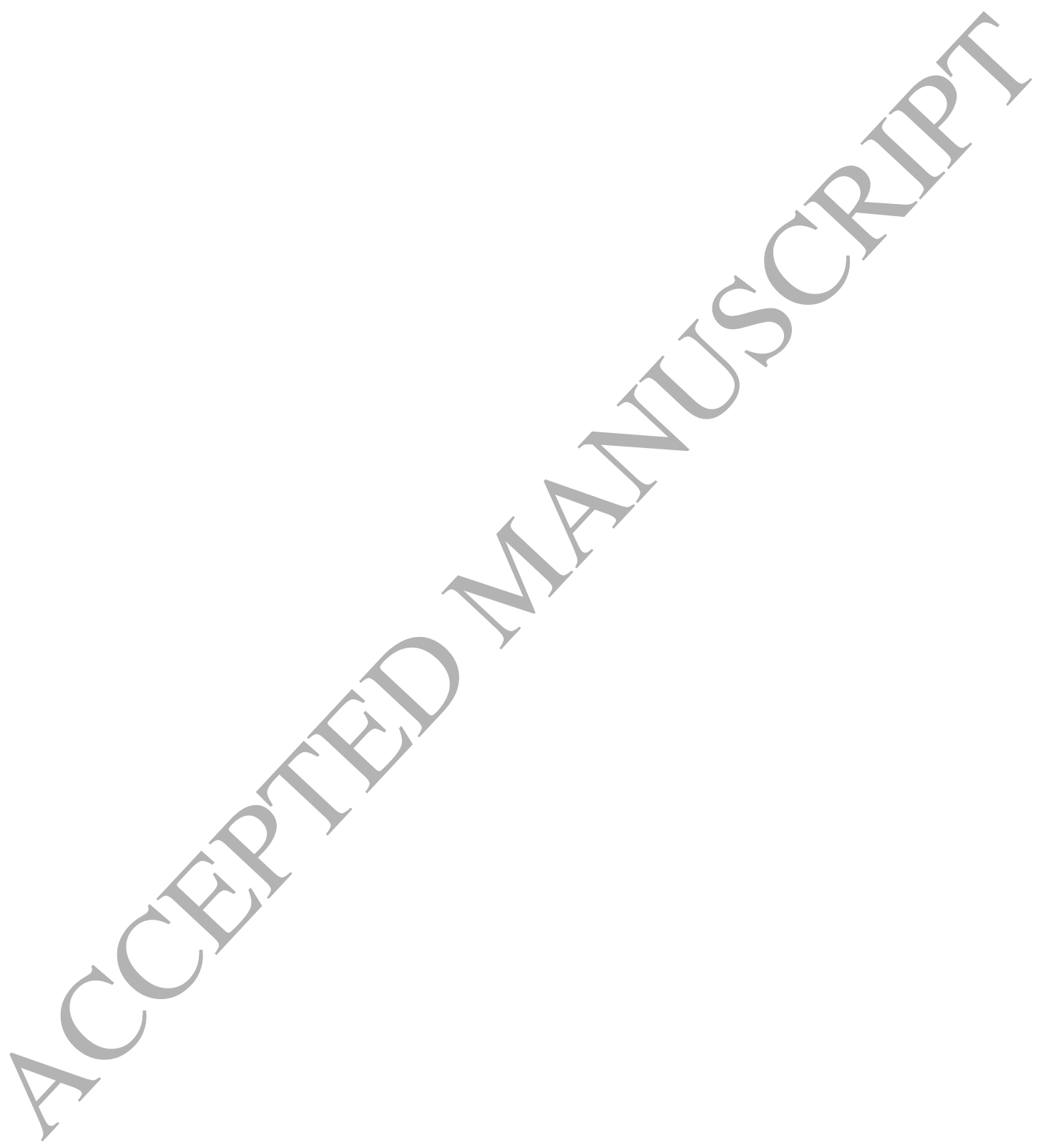




\title{
Finite element modeling effects of inter-yarn friction on the
}

\section{single-layer high-performance fabrics subject to ballistic}

\section{impact}

\author{
Yanyan $\mathrm{Chu}^{1,3,4}$, Xiaogang $\mathrm{Chen}^{1,2}$
}

1. Zhongyuan University of Technology, Zhengzhou, Henan, 450007, China 2. The University of Manchester, Manchester, M13 9PL, UK

3. Collaborative Innovation Centre of Textile and Garment Industry, Zhengzhou, Henan, 450007, China

4. Research Institute of Textile and Garment industry, Zhengzhou, Henan, 450007, Ćnina

\begin{abstract}
This paper aims to numerically figure out the effects of inter-yarn friction in the fabrics impacted by a cylindrical-nose projectile on the ballistic performance including transverse deformation of fabrics, overall energy absorption and the forms of energy absorption. Finite element (FE) models were established for two fabrics based on the yarn properties of Twaron ${ }^{\circledR}$ and Dyneema ${ }^{\circledR}$, respectively. The numerical results show that increasing inter-yarn friction decreases the transverse deflection abilities of the two fabrics and subsequently the response modes of them will transfer from a localized response to a globalized one. With the increase of inter-yarn friction, the energy absorption rate monotonously increases as well while the failure time firstly decreases and then increases but further decreases again. Increasing inter-yarn friction also affect the forms of energy absorption. Near zero friction, strain energy (SE) is the dominant mechanism of a fabric. With the increase of inter-yarn friction, kinetic energy (KE) becomes the dominant one. A maximum inter-yarn friction exists for frictional dissipation energy (FDE) absorption.
\end{abstract}

Keywords: Inter-yarn friction; ballistic impact; transverse deflection ability; fabric response mode; energy absorption. 


\section{Introduction}

With the advancement of technology, fabrics such as para-aramid fabrics and UHMWPE fabrics are more and more used for ballistic protection because of their high strength to weight ratio. Correspondingly, investigations about the behaviours of these fabrics upon ballistic impact attract more attention. Most of researchers begin to explore the relevance between the structure of ballistic fabrics and the ultimate performance of them so as to obtain the optimal design. Nevertheless, the ballistic fabrics are hierarchically constructed where the ballistic fabrics are commonly made from dozens of layers of fabrics and the one layer of fabric is constructed by hundreds of yarns and one yarn is an assembly of thousands of fibres. Given the complexity of hierarchical structure of ballistic fabrics, the influence factors from the structure are considerable $^{[1]}$, for instance, the strength, the pattern, the warp and weft yarn density and so on. Among those numerous influence factors, the inter-yarn friction is outstanding and vital and its effects on the ballistic performance have been investigated by some researchers, such as Hearle et al. ${ }^{[2]}$, Briscoe and Motamedi ${ }^{[3]}$, Duan et al. ${ }^{[4,5]}$, Zeng et al. ${ }^{[6]}$, Rao et al. ${ }^{[7]}$, and Sun et al. ${ }^{[8]}$, Minh et al. ${ }^{[9]}$, Gogineni et $a l .{ }^{[10]}$, Nilakantan et al ${ }^{[11]}$.,Sun et al. ${ }^{[12,13]}$, Zhou et al. ${ }^{[14]}$, Wang et al. ${ }^{[15]}$ and Daset al. [16].

The investigation methods about ballistic impact are mainly limited to numerical method, empirical method and analytical method ${ }^{[17]}$. Empirical method is based on a certain experimental design and device provided to acquire the first-hand data. Through data analyses, the response characteristics of a fabric can be examined and the constitutive relations can be established. However, the disadvantage of this method is obvious for that it is a time consuming and material costly process. Additionally, the accuracy of the obtained results to a large degree depends on the correctness and completeness of collected data. Analytical method is mainly from a physical perspective to set up governing equations based on general mechanic laws using 
various parameters involved in the ballistic impact process. In the analyses, the whole process is divided into $n$ steps by a small increment of time. At the first increment, the equations are usually derived based on energy conservation, impulse theory and Newton's second law. Analogously, step by step, until the ultimate time, all the equations at every time increment are derived. Compared with the empirical method, the analytical method consumes fewer materials and relies on less labour. However, it needs complete understanding of ballistic impact process. Additionally, in order to simplify the process, some of the parameters tend to be neglected. Inter-yarn friction is one of those factors. The truth is that inter-yarn friction plays a significant role in energy absorption during the impact. The numerical investigation is an investigation approach on the basis of the Finite Element (FE) theory and commercial computer software, such as Abaqus, Ansys and LS-DYNA, to establish projectile-fabric simulation model for elucidating the mechanism behind a fabric subjected to impact. FE theory is better suited to analyse dynamic mechanic problems, where a bullet striking a piece of fabric is rightly a dynamic mechanics problem. The conception of FE theory is that the integrity is divided into limited and small units, which is called mesh, the strain and stress and displacement experienced by individual mesh can be computed step by step when loaded. Eventually, varieties of stress and strain taken by a yarn can be acquired. It is an efficient and effective approach because the complex and instantaneous process would be converted into a controllable and visual process through the simulation. Consequently, a large number of researchers started to investigate ballistic behaviours of fabrics using FE simulation.

Due to limited power of computers in previous time, the numerical investigation about the effects of inter-yarn on ballistic performance is investigated under small size. In other words, the sizes of samples in previous simulation work are smaller than those in actual ballistic impact experiment ${ }^{[4-11,14-16]}$. Cunniff $^{[18]}$ applied a sample holder with three sizes of apertures to clamp a single-layer fabric. Apparently, the size of the aperture determines the size of the sample in the test. The ballistic test indicated 
that the residual velocity of the projectile is strongly affected by the sample holder aperture size at impact velocities near the ballistic limit of the fabric. In this velocity range, the smaller the aperture is, the higher the residual velocity is.

According to Cunniff's investigation, neglecting the effects of the sample size in the previous studies may lead to incorrect understanding because the sample size did have effects on ballistic performance. In the past, we have established a full size model to analyse the effects of inter-yarn ${ }^{[19]}$. The effects of inter-yarn friction on fabric failure mechanism have been comprehensively elucidated from perspectives of the role of primary yarns, and secondary yarns. In this study, we will further dig out the effects of inter-yarn friction on the ballistic performance of fabrics with respect to the energy absorption behaviour and fabric response modes.

\section{Simulation work}

\subsection{The modelling}

In modelling part, the task is to simulate a ballistic perforation process that one layer fabric is impacted by a cylindrical nose projectile. The fabric size in current model is the same as the actual size used for ballistic impact test, which is a simple plain fabric. It is constructed from yarns with the linear density of 93 tex. The warp and weft density are designed to be identical, 7.8 ends/cm. The areal density of the fabric is $153.61 \mathrm{~g} / \mathrm{cm}^{2}$. It is circle and the radius of it is $7.5 \mathrm{~cm}$. Since the projectile-fabric assembly is symmetric, only a quarter of the fabric area is needed for the simulation. In the simulation, the average width of the cross-section of the yarn in model is 1.134 $\mathrm{mm}$ and the average wavelength of the yarn is $2.556 \mathrm{~mm}$. The average thickness of the fabric is measured as $0.210 \mathrm{~mm}$. The projectile is a steel cylinder with the diameter and height both being $5.5 \mathrm{~mm}$. The mass of it is $1.00 \mathrm{~g}$. The yarn and the projectile are meshed by C3D8R elements and the cross-section of the yarn is meshed with ten elements, as shown in Figure 1. The quarter fabric described in Figure 2 is drawn in $\mathrm{x}-\mathrm{Z}$ plane, where the circumference of it is fixed, and the remaining two sides are 
given symmetric boundary condition. The projectile is only allowed to move along the negative direction of $\mathrm{Y}$ axis. The validation of $\mathrm{FE}$ modelling results is accomplished through comparing the residual velocity and the transverse wave velocity from the FE results to the ones from the ballistic test results at the similar impact velocity. The validation process can refer to our previous work ${ }^{[19]}$.

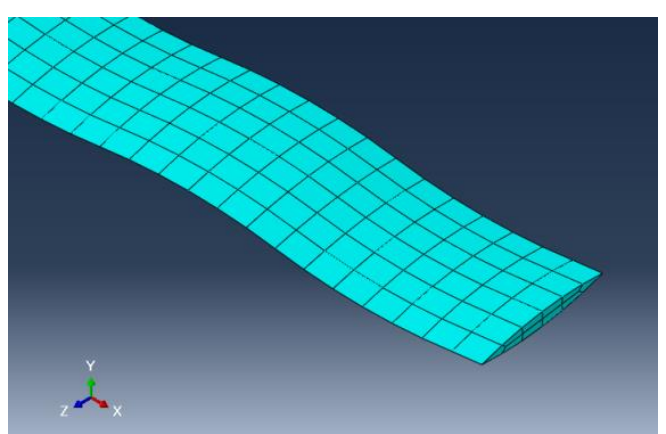

(a) The yarns

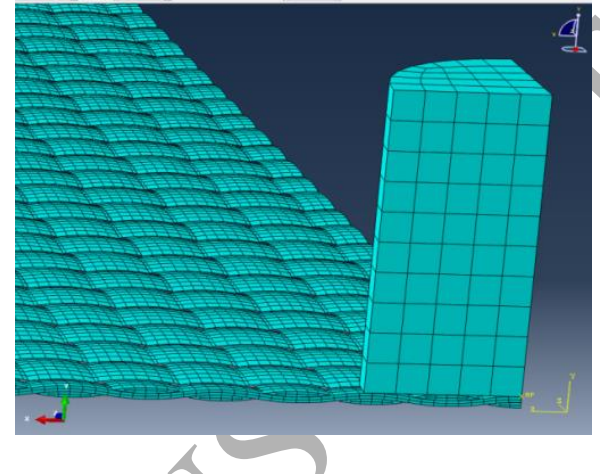

(b) the whole systerm

Figure 1 Mesh image of a layer fabric with projectile

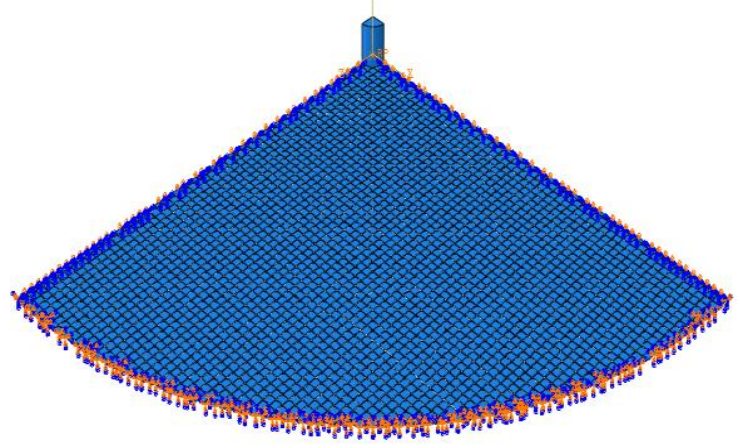

Figure 2 Boundary conditions used in the model

\subsection{The materials and impact parameters}

The material constants for the yarns are dominated by the longitudinal tensile properties although the real yarns are made of bundled fibres with orthotropic properties. As such, the yarns are assumed to be homogeneous and isotropic ${ }^{[4-5,7,21]}$. Previous researchers have shown that the homogeneity and isotropy assumptions of the yarn lead to acceptable results with inaccuracy approximately $2.4 \%$ in energy absorption $^{[22-23]}$. The effects of inter-yarn friction will be investigated under two Young's moduli conditions, one being $72 \mathrm{GPa}$ and the other being $112 \mathrm{GPa}$. The 72 GPa is set according to the Young's modulus of Twaron ${ }^{\circledR}$ yarns. Based on previous 
investigations ${ }^{[6]}$, the Young's modulus of Twaron ${ }^{\circledR}$ yarns is $72 \mathrm{GPa}$ at the strain rate of 1000 /s. The $112 \mathrm{GPa}$ is set according to the Young's modulus of Dyneema ${ }^{\circledR}$ yarns. In the research from Koh et al. ${ }^{[24]}$ and Huang et al. ${ }^{[25]}$, the Young's modulus of Dyneema $^{\circledR}$ yarn is around $80 \mathrm{GPa}$ at the strain rate around $1000 \mathrm{~m} / \mathrm{s}$ while from the tests carried out by Russell et al. ${ }^{[26]}$, the Young's modulus of Dyneema ${ }^{\circledR}$ is around 130 $\mathrm{GPa}$ at the same strain rate range. Russell et al. explained that the discrepancy is associated with the measurement method and the slip between specimen and grips ${ }^{[26]}$. Form other references ${ }^{[27-29]}$, the Young's modulus of Dyneema ${ }^{\circledR}$ yarns is around 107-115 GPa. Thus, the Young's modulus of Dyneema ${ }^{\circledR}$ yarns is set as $112 \mathrm{GPa}$ at the high strain rate of 1000/s. For the yarns, the deformation of the yarn prior to reaching the yield point creates only elastic strain, which are fully recovered if the applied load is removed. However, once the stress in the yarns exceeds the yield stress, permanent plastic deformation begins to occur, the strains associated with this permanent deformation are called plastic strains. Based on our test and previous investigations, these two yarns almost keep elastic linear property until failure. Therefore, the elastic strain is set as 0.0428 and the plastic strain is set smaller as 0.001. For the DYM model, the elastic strain and the plastic strain are same to the TM model. Since its modulus is $112 \mathrm{Gpa}$, therefore, its failure stress $\sigma$ should be larger and it is $5.11 \mathrm{Gpa}$, which is calculated from the following formula (1) by reference document of Getting Started with Abaqus.

$$
\sigma=\sigma_{\text {nom }}\left(1+\varepsilon_{\text {nom }}\right)
$$

$\varepsilon_{\text {nom }}$ is the sum of elastic strain and the plastic strain. $\sigma_{\text {nom }}$ is the product of the elastic Young's modulus and the elastic strain.

In order to compare the two model, the other material properties in the two moduli cases are kept same and set according to Twaron ${ }^{\circledR}$ yarns although the density for Dyneema $^{\circledR}$ yarn is different from Twaron $^{\circledR}$. The density is set as $1268 \mathrm{~kg} / \mathrm{m}^{3}$ according to the packing density of Twaron $^{\circledR}$ fibres in yarns with a value of 
approximately 0.89 . The Poisson ratio of the yarn is set as $0.3^{[10]}$. These two moduli cases are named Twaron ${ }^{\circledR}$ fabric model $(\mathrm{TM})$ and Dyneema ${ }^{\circledR}$ yarn based model (DYM), respectively.

A progressive damage model which reflects the material property degradation is implemented in ABAQUS/ Explicit to allow the fracture of the material ${ }^{[30]}$. The simulation of material failure consists of three stages: (i) establishment of the damage initiation criterion, (ii) determination of the damage evolution law and (iii) element removal upon reaching a completely damaged state. The failure of the material is usually divided into brittle failure or ductile failure. The failure mode of yarns in the fabric is close to stress-based ductile damage criterion. Sun et al. ${ }^{[23]}$ and Wang et al. ${ }^{[22]}$ used this damage criterion in their simulation analysis. The damage initiates when the condition in formula (2) is satisfied:

$$
\begin{aligned}
\mathrm{w}_{\mathrm{D}} & =\int \frac{\mathrm{d} \bar{\varepsilon}^{\mathrm{pl}}}{\bar{\varepsilon}_{\mathrm{D}}^{\mathrm{pl}}\left(\gamma, \dot{\bar{\varepsilon}}^{\mathrm{p}}\right)}=1 \\
\gamma & =-\mathrm{p} / \mathrm{q}
\end{aligned}
$$

Where $w_{D}$ is the state variable, $\bar{\varepsilon}^{\mathrm{pl}}$ is equivalent plastic strain. $\bar{\varepsilon}_{\mathrm{D}}^{\mathrm{pl}}$ is a function of stress triaxiality $\gamma$ and strain rate., The method for computing triaxiality $\gamma$ is shown in formula $(3)^{[30]} \cdot \mathrm{p}$ is the pressure stress, $\mathrm{q}$ is the Mises equivalent stress. $\dot{\bar{\varepsilon}}^{\mathrm{pl}}$ is the equivalent of plastic strain rate.

When the material damage occurs, the stress-strain relationship no longer accurately represents the material's behaviour. Hillerborg et al.'s fracture energy proposal is then used to reduce mesh dependency by creating a stress-energy response after damage initiation to define the damage evolution. Hillerborg et al. ${ }^{[31]}$ defined the energy, $\mathrm{G}_{\mathrm{f}}$, as a material parameter. It indicates the required energy to open a unit area of a crack and is given in formula (4). Removal of a solid element is set by default, when maximum degradation is reached at any one integration point.

$$
\mathrm{G}_{\mathrm{f}}=\int_{\varepsilon_{o}^{p l}}^{-\overline{\varepsilon_{f}}} L \sigma_{y} d \bar{\varepsilon}^{p l}=\int_{0}^{\bar{u}^{p l}} \sigma_{y} d \bar{u}^{p l}
$$


Where $\mathrm{L}$ is the characteristic length of the mesh; $\overline{\mathrm{u}}^{\mathrm{pl}}$ is the equivalent plastic displacement, equalling to the product of $\mathrm{L}$ and $\dot{\bar{\varepsilon}}^{\mathrm{pl}} ; \sigma_{\mathrm{y}}$ is the yield stress.

The projectile is steel with the density of $7687 \mathrm{~g} / \mathrm{cm}^{3}$. Compared with the fabric, the projectile is much stiffer. As such, the projectile is defined as a rigid body, which means that it is not deformable in the whole process. The Poisson ratio is set as $0.3^{[32]}$. A pair of coefficients of friction including coefficient of static friction (CSF) and coefficient of kinetic friction (CKF) is set to indicate the variations of inter-yarn friction in the fabric. The CSF varies from 0 to 1 and CKF from 0.05 to 0.95 with 0.1 intervals. The impact velocity of the projectile in the FE simulation is set as $475 \mathrm{~m} / \mathrm{s}$ since it is averaged around $475 \mathrm{~m} / \mathrm{s}$ in the ballistic experiment.

\subsection{The computation of the overall energy absorption}

The energy absorption computed in the following analyses is based on the entire fabric although the model in FE simulation is a quarter based. In the simulation of perforation test, the impact velocity and the residual velocity can be measured. Thus, variation in projectile energy loss can be calculated from these two velocities ${ }^{[33-37]}$, as shown in formula (5). Without any other external force action and without considering energy loss by heat, intermolecular friction, air resistance, acoustic energy and so on, the projectile energy loss can be assumed to be fully absorbed by the fabric based on the law of energy conservation. The energy transfer between the projectile and fabric can be simply described in the following formula (6):

$$
\begin{gathered}
\Delta E_{p}=\frac{1}{2} m\left(v_{i}^{2}-v_{r}^{2}\right) \\
\Delta E_{p}=\Delta E_{f}
\end{gathered}
$$

where $\Delta E_{p}$ is projectile energy loss; $m$ is the mass of a projectile; $v_{i}$ is impact velocity, and $v_{r}$ is residual velocity; $\Delta E_{f}$ means overall energy absorption of a fabric.

\section{Results and discussion}




\subsection{The effects of inter-yarn friction on transverse deformation of the fabric}

\subsubsection{Transverse deflection ability}

At the end of ballistic impact, a pyramid-like shape would form on the back face of the fabric due to the transverse deflection. After impact, an angle $\beta$ has been formed between the deformed fabric and the undeformed one, as shown in Figure 3, which equals to the arc tangent value of the ratio between back face signature(BFS) depth $D$ and BFS base radius $R$, referring to formula (7). A smaller angle $\beta$ is desirable because smaller angle means that the fabric is less prone to deflect transversely upon the ballistic impact and thus cause fewer traumas.

$$
\beta=\tan ^{-1} \frac{D}{R}
$$
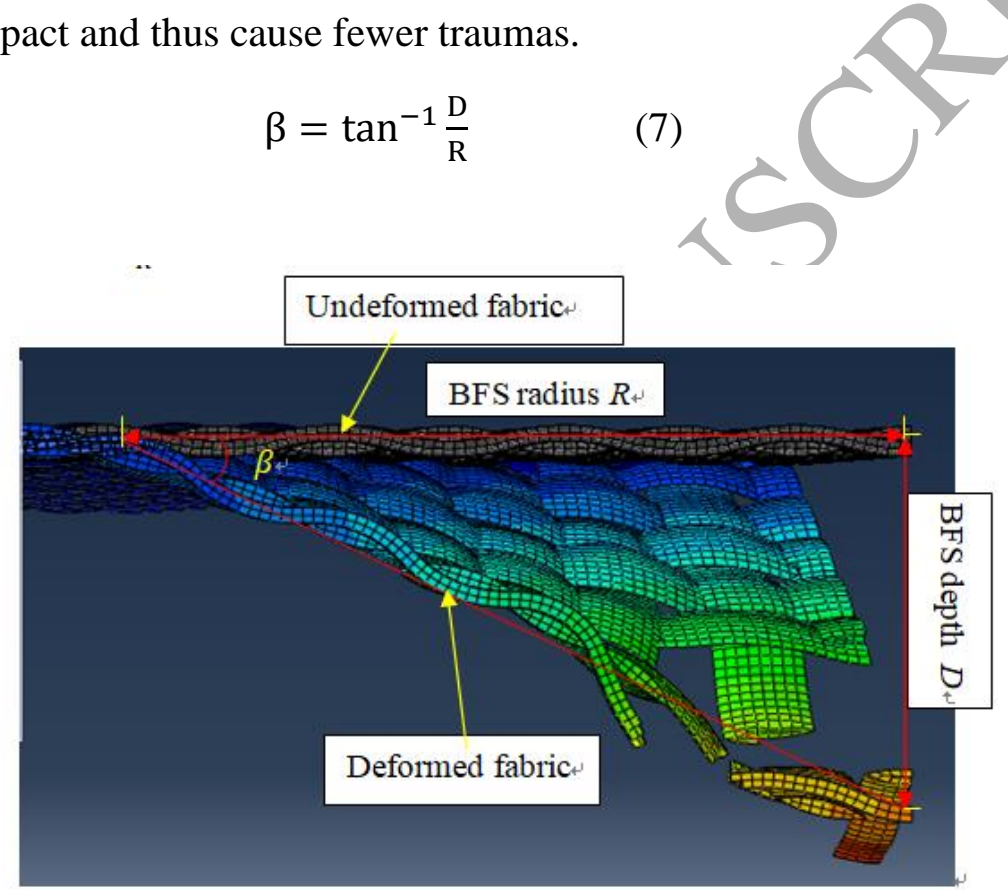

Figure 3 The illustration of the fabric deformation

Figure 4 shows the trend of $\beta$ with the increase of inter-yarn friction. It shows that the angle $\beta$ decreases with the inter-yarn friction increasing from zero friction to CSF of 0.40 and $\mathrm{CKF}$ of 0.35 . It is assumed that more inter-yarn friction at crossover may restrain the bending of the yarn and thus the fabric transversely deflects less. After a certain level of inter-yarn friction, the transverse deflection ability is no longer affected by the inter-yarn friction and the materials, where the explanation may be that constrains from the friction at the crossover reaches the maximum and over high inter-yarn friction cannot take any effect. 


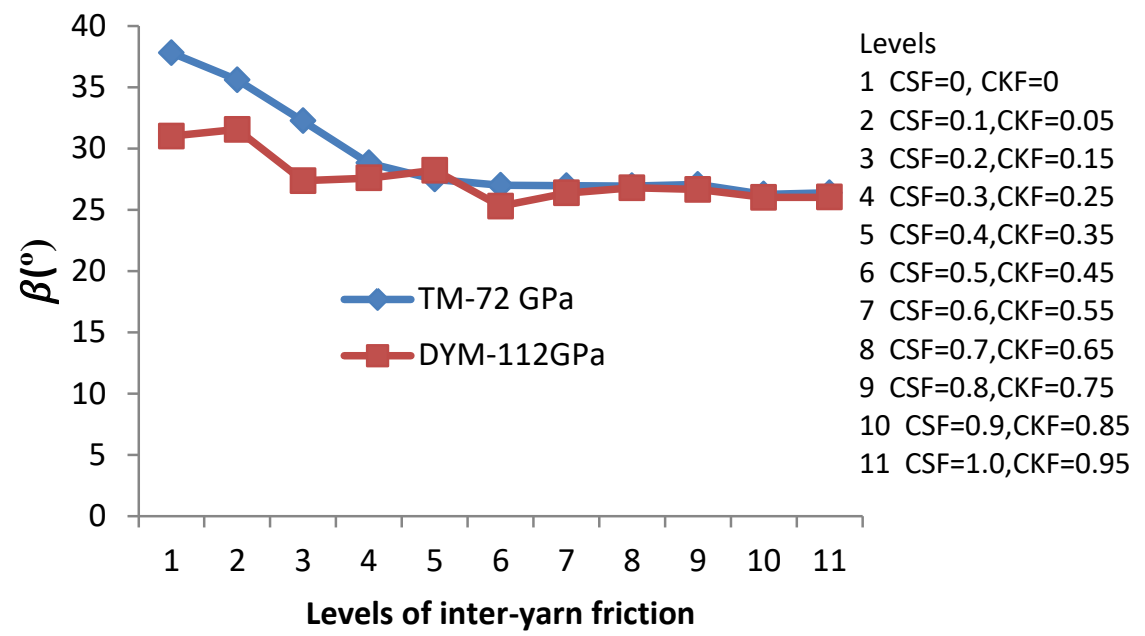

Figure 4 The $\beta$ as the function of inter-yarn friction

In addition, in the low frictional range, increasing inter-yarn friction will more significantly decrease the transverse deflection ability of the TM fabric than that of the DYM fabric. The explanations are as follows. The transverse deflection ability of the fabric is related to the transverse modulus of the yarn in it. For the transverse modulus, it is positively dependent on the Young's modulus of the yarn and thus the transverse modulus of Dyneema ${ }^{\circledR}$ yarn is larger than that of Twaron ${ }^{\circledR}$ yarn. Since the transverse deflection ability of the fabric is related to the transverse modulus of the yarn, where the larger transverse modulus of the yarn means the corresponding fabric is less likely to deflect transversely, the transverse deflection ability of the DYM fabric is smaller than that of the TM fabric and consequently the room for decreasing the transyerse deflection ability of the DYM fabric is less. Hence, the transverse deflection ability of the TM fabric will be more significantly affected with the initial increase of inter-yarn friction than that of the DYM fabric.

\subsubsection{The fabric transverse response modes}

In the ballistic impact, it is more anticipated that the fabric gives a globalized response rather than a localized one because the localized response would cause more serious trauma. The illustration of the localized response and the globalized one are 
shown in Figure 5(a). A localized response means that the whole projectile energy distributes on a small area but the globalized response indicates that the energy spreads through a larger area of the fabric. To investigate the effect of inter-yarn friction on the response modes, the effects of inter-yarn friction on the back face signature are firstly plotted in Figure 5 (b) and (c). Those curves were plotted using the position of primary yarns in the fabric at the moment when it fails. It was observed that the back face signature will become smaller as the inter-yarn friction increases to CSF of 0.5 and CKF of 0.45 in TM case and CSF of 0.3 and CKF of 0.25 in DYM case. Beyond those levels of inter-yarn friction, the back face signature inversely increases.
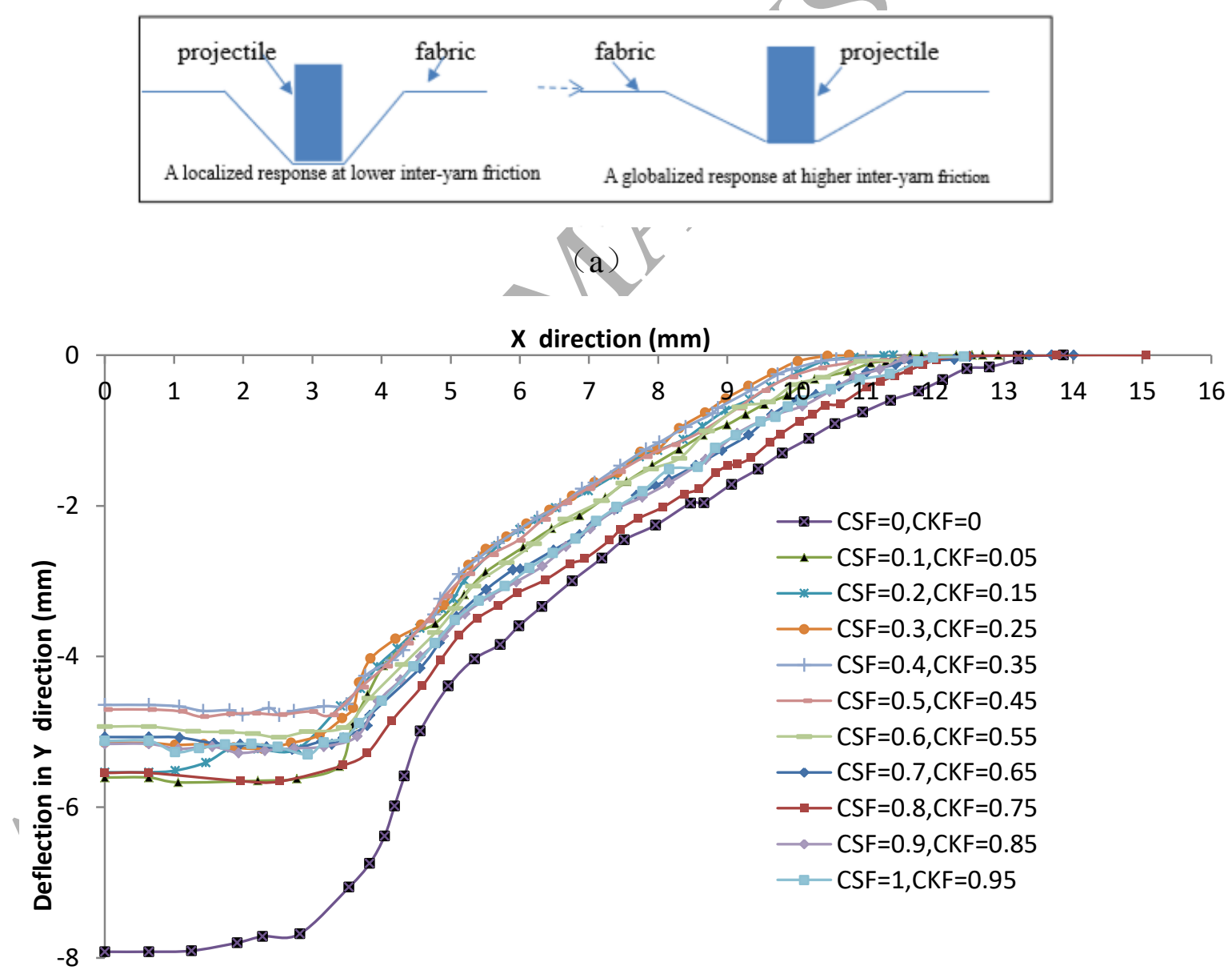

(b) 


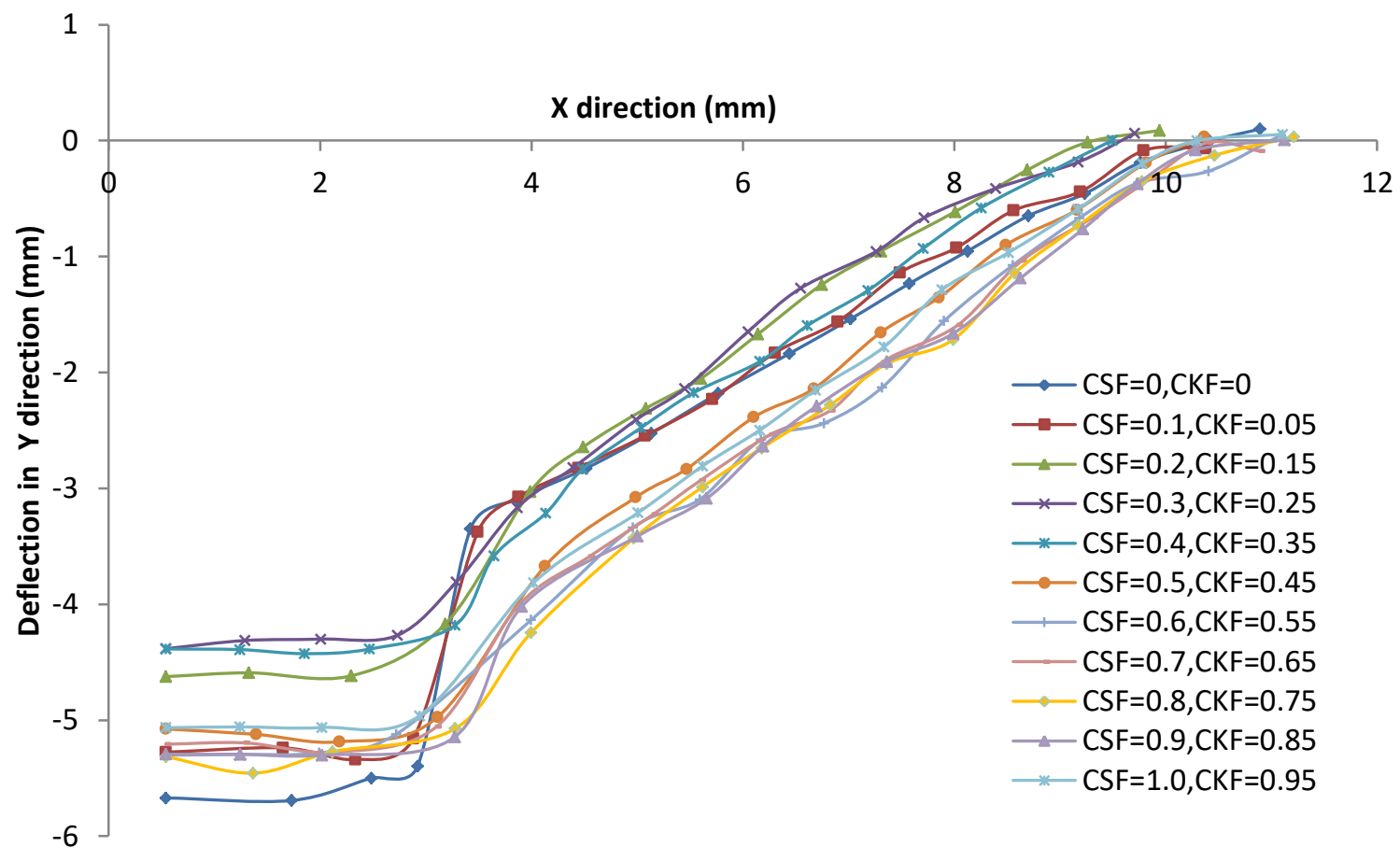

(c)

Figure 5 The fabric response mode, (a) the localized response mode and the globalized response mode. (b) the back face signatures of TM fabrics with different inter-yarn friction levels; (c)the back face signatures of DYM fabrics with different inter-yarn friction levels;

Since the final back face signature depends on the failure time, taking BFS depth $D$ and BFS base radius $R$ to be divided by the failure time $t$ individually, as displayed in Formulas (8) and (9), two speeds $\mathrm{u}_{\mathrm{lab}}$ and $V_{d}$ corresponding to the transverse wave velocity and the transverse deflection velocity are obtained, referring to Figure 6 . The transverse deflection/velocity and the transverse wave velocity can be taken into account because the transverse wave velocity and transverse deflection velocity indicate the distribution rate of energy in horizontal and vertical direction respectively. Figure 7 and 8 individually show the transverse deflection velocity and transverse waye velocity as function of inter-yarn friction. It can be seen that increasing inter-yarn friction to the level of CSF of 0.5 and CKF of 0.45 is better for decreasing the transverse deflection velocity and accelerating the transverse wave velocity. In other words, increasing inter-yarn friction will cause the response of the fabric transfer from a more localized one to globalized one. 


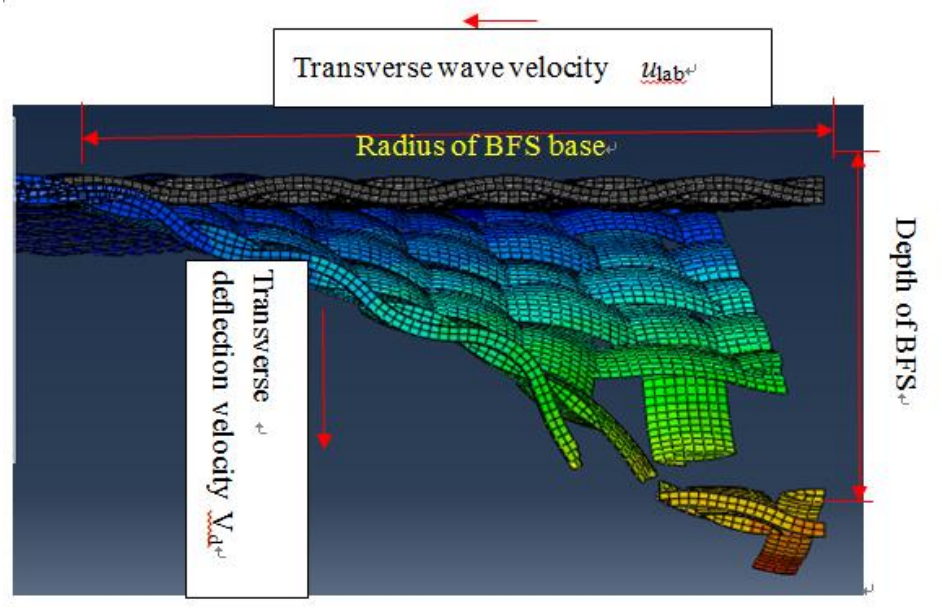

Figure 6 the trasverse wave velocity and the deflection velocity

$$
\begin{aligned}
& \mathrm{V}_{\mathrm{d}}=\frac{\mathrm{D}}{\mathrm{t}} \\
& \mathrm{u}_{\mathrm{lab}}=\frac{\mathrm{R}}{\mathrm{t}}
\end{aligned}
$$

(9)

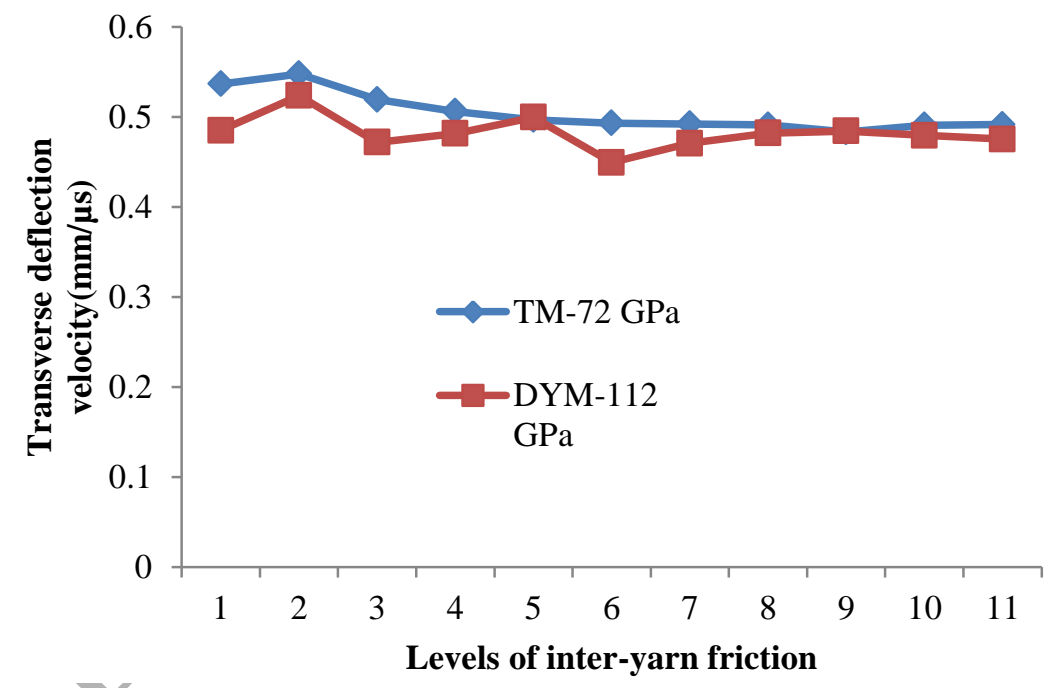

Figure 7 The effects of inter-yarn friction on the transverse deflection velocity 


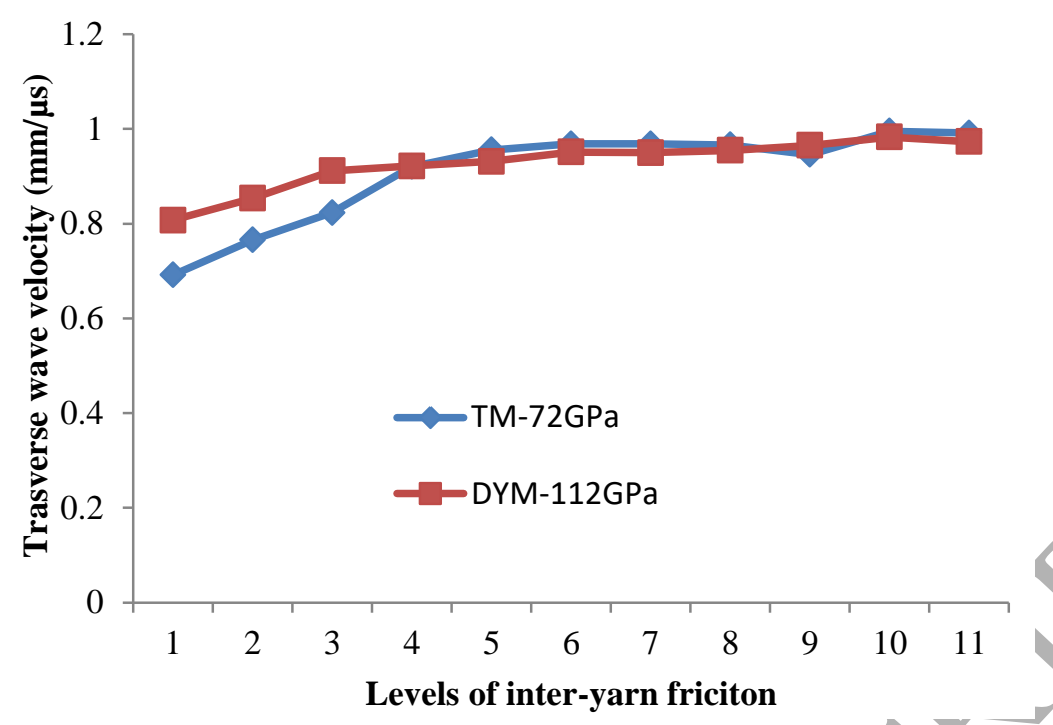

Figure 8 The effects of inter-yarn friction on the transverse wave velocity

\subsection{Effects of inter-yarn friction on energy absorption}

\subsubsection{Overall energy absorption}

The overall energy absorption is the total energy absorbed by the fabric ultimately, which equals to the total projectile energy loss. The changing trend of overall energy absorption with the increase of inter-yarn friction is plotted in Figure 9. There are some similarities in the two moduli cases. The overall energy absorption presents an increasing trend as the level of the inter-yarn friction increases from CSF of 0.30 and CKF of 0.25 to CSF of 0.80 and CKF of 0.75 in case of TM and to CSF of 0.60 and CKF of 0.55 in case of DYM and it slightly decreases at much higher inter-yarn friction. The difference between the two cases lies in the levels of the inter-yarn friction from zero to CSF of 0.30 and CKF of 0.25 . In this range, the overall energy absorption/in the DYM 112 GPa case gradually grows with the increase of inter-yarn friction but it shows an opposite trend in the TM 72 GPa case. It should be noted that the overall energy absorption in DYM case is larger than that in TM case because larger modulus is more efficient in dissipating the impact energy, leading to more energy absorption. 


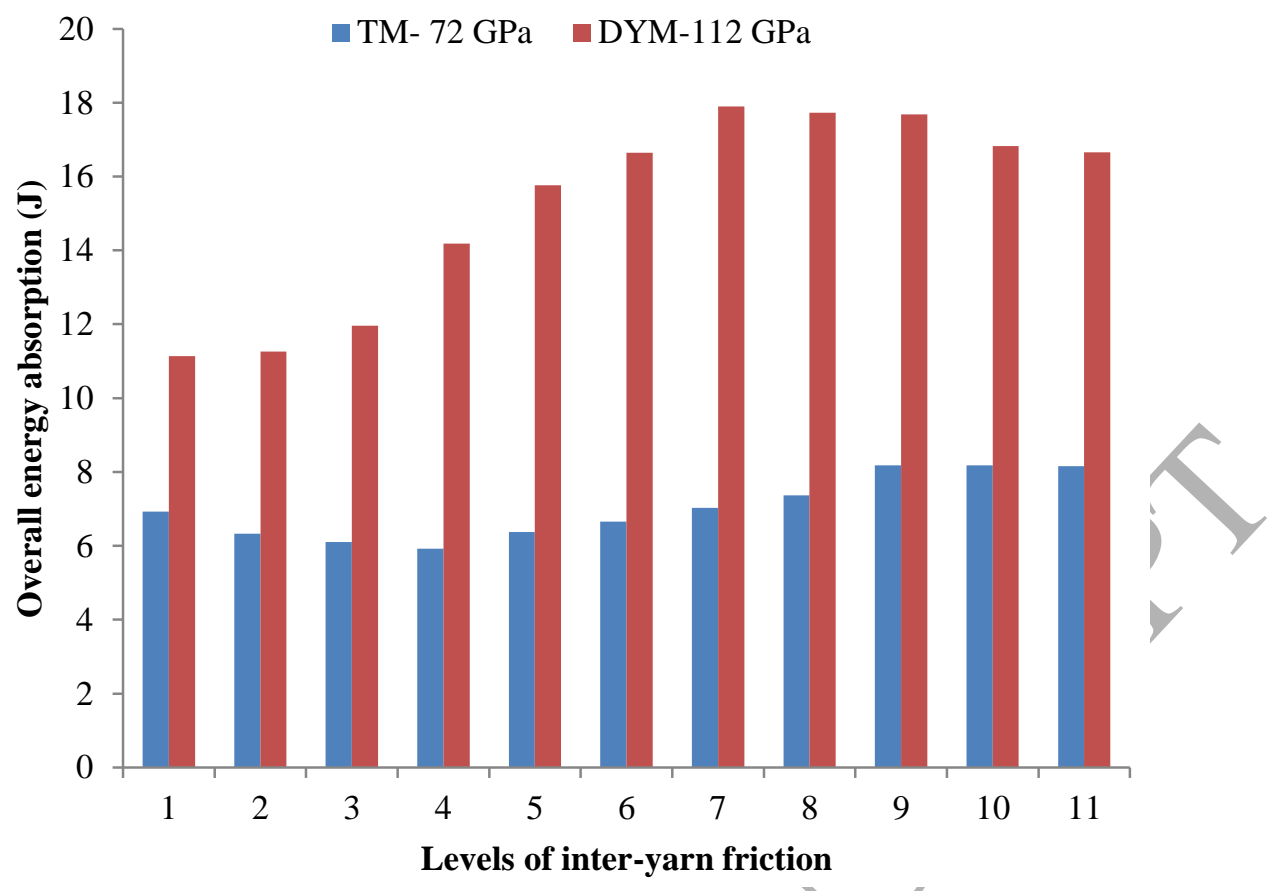

Figure 9Overall energy absorption with increase of inter-yarn friction

The trend of the overall energy absorption with the increase of inter-yarn friction in DYM $112 \mathrm{GPa}$ case agrees with the previous work carried out by Briscoe and Motamedi $^{[2]}$, Duan et al..$^{[4]}$, Sun et al. ${ }^{[12]}$ and Zhou et al. ${ }^{[14]}$. However, the trend of overall energy absorption decreasing as the inter-yarn friction grows from zero to CSF of 0.30 and CKF of 0.25 has never been found in any previous investigations. It is assumed that the Young's modulus plays a role. Thus, the Young's modulus is extended to a larger range and the trends of overall energy absorption with the increase of inter-yarn friction at those Young's modulus conditions are plotted in Figure 10. When the Young's modulus is relative lower, for instance, $72 \mathrm{GPa}$ and 52 GPa, the trends of the overall energy absorption with the increase of inter-yarn friction are almost similar, which indicates that inter-yarn friction near zero may give rise to higher energy absorption. Since the overall energy absorption is related to the energy absorption rate and the failure time, the effects of inter-yarn friction on these two aspects will be discussed. 


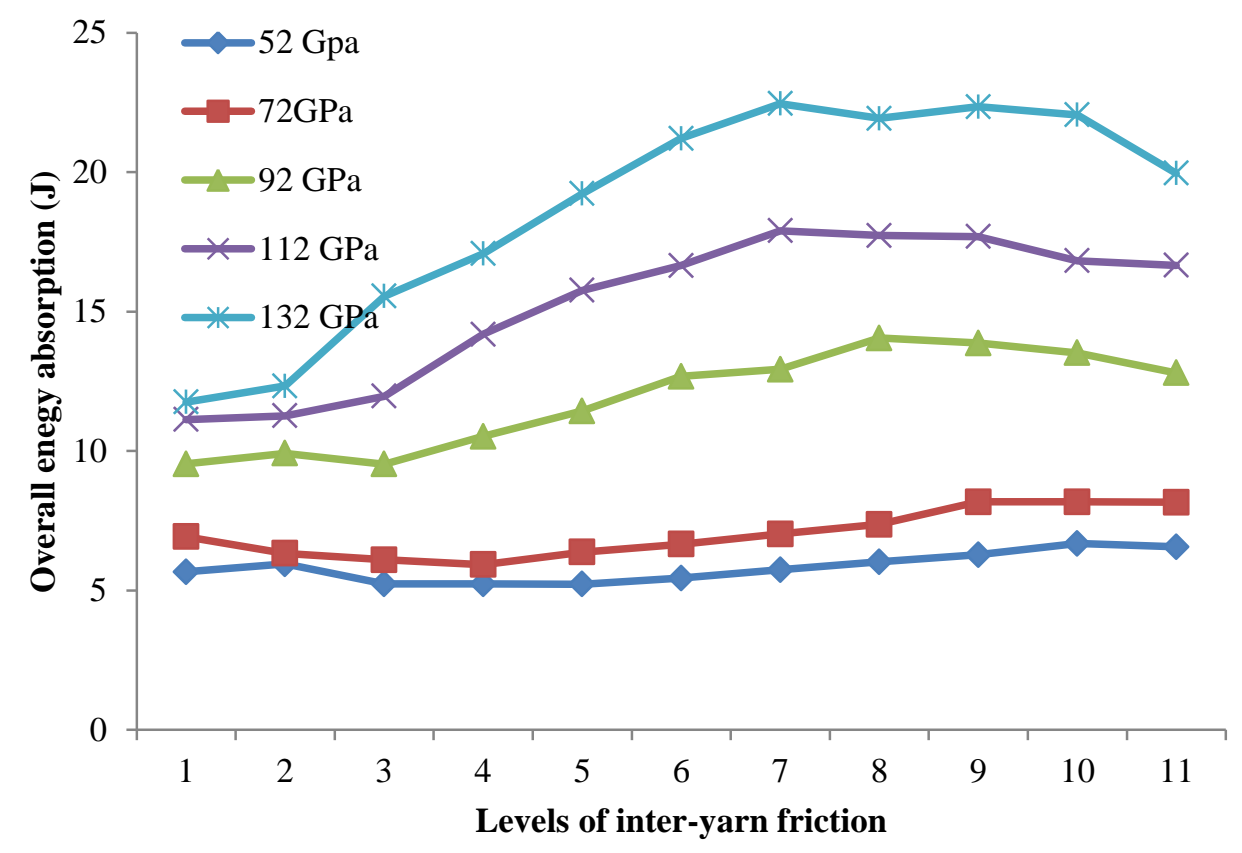

Figure 10 Overall energy absorption at different Young's moduli cases

\subsubsection{Energy absorption rate}

Although the ballistic impact is almost a transient process, the energy absorption is still a time dependent parameter. The energy absorption rate is a parameter computed from the overall energy absorption divided by time. Thus, the energy absorption rate denotes the average energy absorbed in a unit time, or termed as average energy absorption rate which reflects how fast the fabric can dissipate the impact energy. In fact, the energy absorption in the whole time history is not a uniform process. It may be due to the non-homogeneous nature of the fabric structure and the asynchronism of the failure of each yarn. Despite the non-uniformity of the energy absorption, the average energy) absorption rate can still reflect the efficiency of the energy absorption at different frictional levels. The energy absorption rates in TM 72 GPa and DYM 112 GPa cases are plotted as a bar chart in Figure 11. They display a similar pattern with the increase of the inter-yarn friction in these two moduli cases, where it increases monotonously with the increase of inter-yarn friction. Comparing the effect of inter-yarn friction on the overall energy absorption with that on the energy absorption rate, it is clear that the parameter which inter-yarn friction can make increase monotonously is the energy absorption rate rather than the overall energy absorption. 
The following is to analyse the underlying reasons.

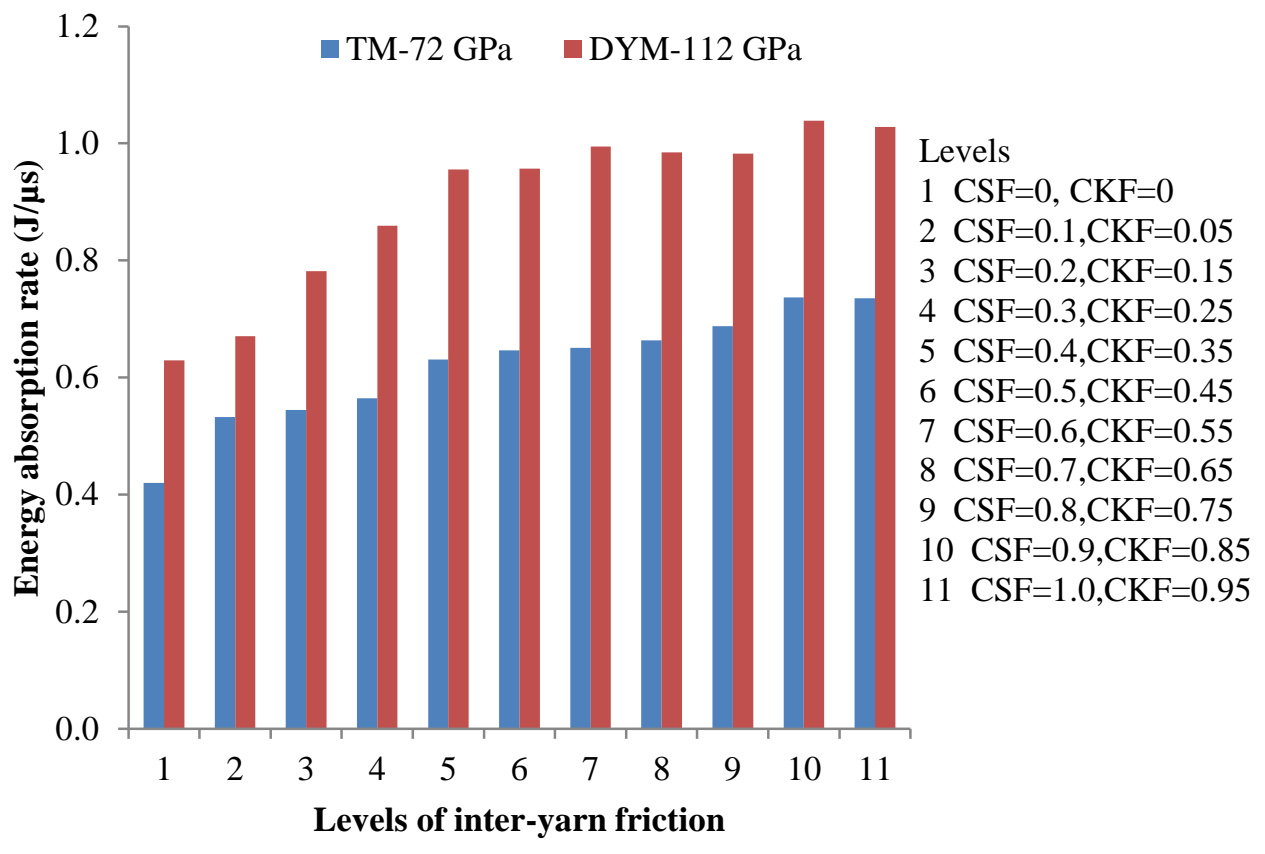

Figure11 The average energy absorption rate with the increase of the inter-yarn friction

Figure 11 indicates that higher inter-yarn friction will result in higher energy absorption rate in the two cases. It may be attributed to the increased resistant forces acting on the projectile from the fabrics with higher friction levels. The higher resistant force from the fabric would accelerate the loss of the projectile velocity and in turn make the energy transfer to the fabric more efficiently. The resistant force can be calculated based on the impulse principle as shown in formula (10). Figure 12 plots the resistant forces acting on the projectile in different frictional levels in TM $72 \mathrm{GPa}$ case and DYM $112 \mathrm{GPa}$ cases, respectively. The resistant force calculated is the average force resisting the projectile during the whole time history. As the process proceeds, the resistant force to the projectile becomes less and less because the fabric structure begins to fail. Despite the resistant force attenuating with time, the average resistant force is still a useful indicator that can assist in reflecting the differences among the resistant forces acting on the projectile. In those two cases, the resistant force on the projectile is higher with higher levels of inter-yarn friction. The reason may be that higher inter-yarn friction makes yarn-yarn joint force at the crossover 
within the fabric become stronger, and thus enables the yarns in the entire fabric function more efficiently to obstruct the projectile.

$$
f t=m v_{i}-m v_{r}
$$

Where $\mathrm{f}$ is resistant force; $\mathrm{t}$ is failure time; $\mathrm{m}$ is the mass of the projectile; $\mathrm{v}_{\mathrm{i}}$ and $\mathrm{v}_{\mathrm{r}}$ are the impact velocity and the residual velocity of the projectile.

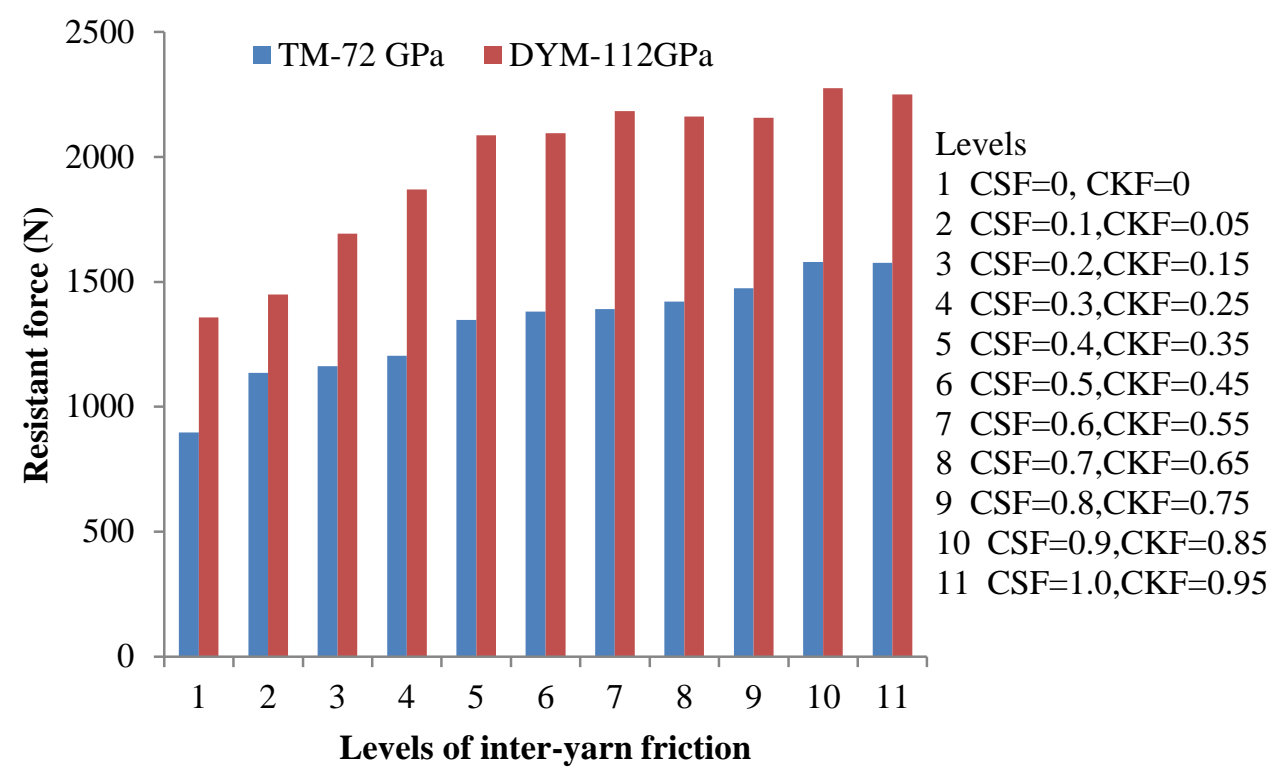

Figure 12 The average resistant force acting on the projectile from the fabric

\subsubsection{The failure time}

The trend of the failure time for the fabric with the increase of inter-yarn friction is shown in Figure 13. The failure time is the total time from the beginning of the impact to the fabric failure, which is the moment when the energy absorbed by the fabric keeps unchanged. In the TM 72 GPa case, the time significantly shortens with the increase of the friction while it becomes a little longer as the CSF grows starting from 0.40 to 0.80 and CKF from 0.35 to 0.75 . When the friction is higher than CSF of 0.80 and CKF of 0.75 , the time becomes shorter once more. As DYM $112 \mathrm{GPa}$ is concerned, the time firstly decreases as the inter-yarn friction increases by CSF of 0.20 and CKF of 0.15 and then gradually increases as the inter-yarn friction increases. When the inter-yarn friction adds up to much higher level such as CSF of 0.80 and 
CKF of 0.75 , the time decreases again. The trends of the failure time with inter-yarn friction in the two cases are similar. The difference between the maximum failure time and minimum is around $2 \mu \mathrm{s}$ in DYM $112 \mathrm{GPa}$ case whilst that for TM is approximate $6 \mu \mathrm{s}$. The descending degree of the failure time with increasing inter-yarn friction corresponds to the descending degree of transverse deflection ability. Therefore, the failure time of the DYM fabric decreases less than that of the TM fabric. The failure mechanism for the two moduli cases can refer to our previous paper ${ }^{[19]}$.

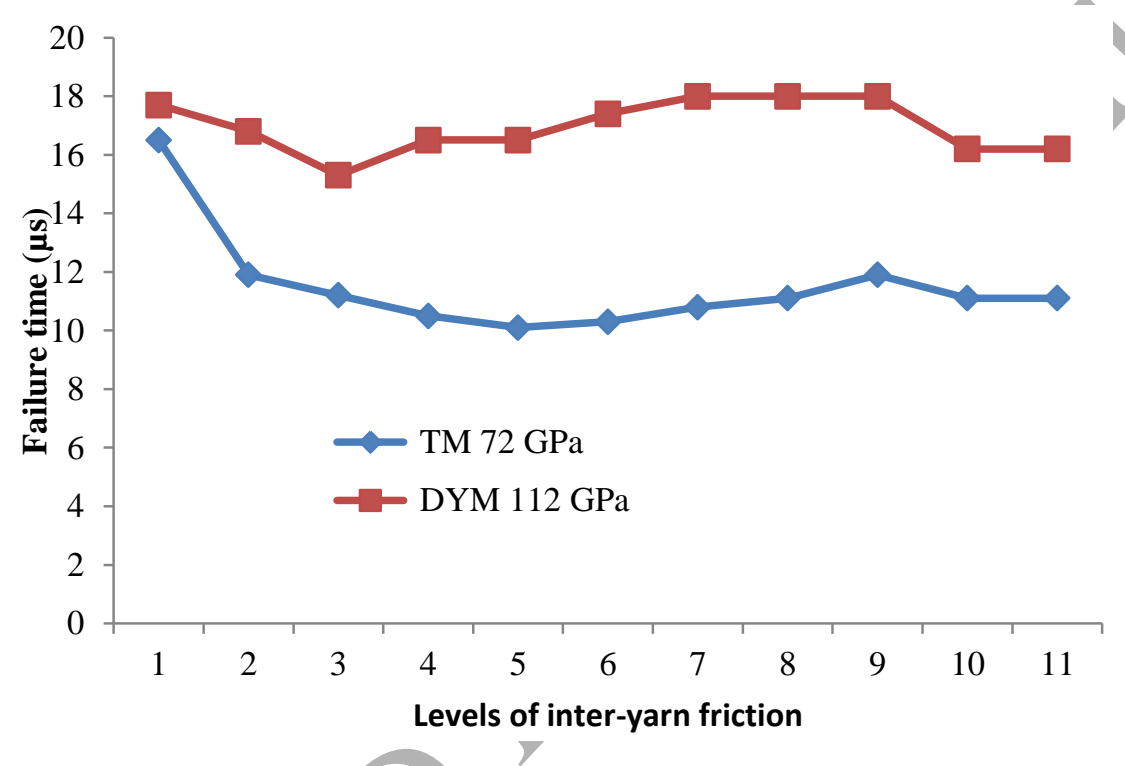

Figure 13The trends of failure time of fabrics with the increase of the inter-yarn friction

\subsubsection{The analyses and discussion on the mechanism of overall energy absorption}

The overall energy absorption depends on two parameters, one being energy absorption rate and the other being total failure time. Increasing overall energy absorption can/be achieved in two manners, absorbing energy much more efficiently and prolonging the failure of the fabric. However, in the previous investigations, researchers only observed the effects of inter-yarn friction on either energy absorption rate or failure time ${ }^{[4-14]}$. On one hand, slightly increasing the inter-yarn friction will result in higher energy absorption rate owing to the higher resistance force; On the other hand, it would aggravate the stress concentration from tension stress and shear stress acting on the primary yarns near the impact area and decrease the transverse deformation ability, leading to less failure time ${ }^{[19]}$. 
The curves about time history of energy absorption at different levels of inter-yarn friction are plotted below in Figure 14 and 15. The slope of the curve represents the energy absorption rate and the moment when the curve becomes flatten indicates the failure of the fabric. It is not difficult to discover that the overall energy absorption with the increase of inter-yarn friction in the TM case is more dependent on the failure time in the frictional range from zero friction to CSF of 0.30 and CKF of 0.25 ; while from CSF of 0.30 and CKF of 0.25 to CKF of 0.80 and CSF of 0.75 , it is more dependent on the energy absorption rate. However, it should be noted that although the TM fabric absorbs more energy at zero friction, the energy absorption mode is not desirable because it is a localized energy absorption mode. In DYM case, the overall energy absorption more relies on the energy absorption rate from zero friction to frictional level of CKF of 0.80 and CSF of 0.75. At higher levels of the inter-yarn friction, the overall energy absorption in both moduli cases begins to decrease because the failure time decrease again, referring to Figure 13.

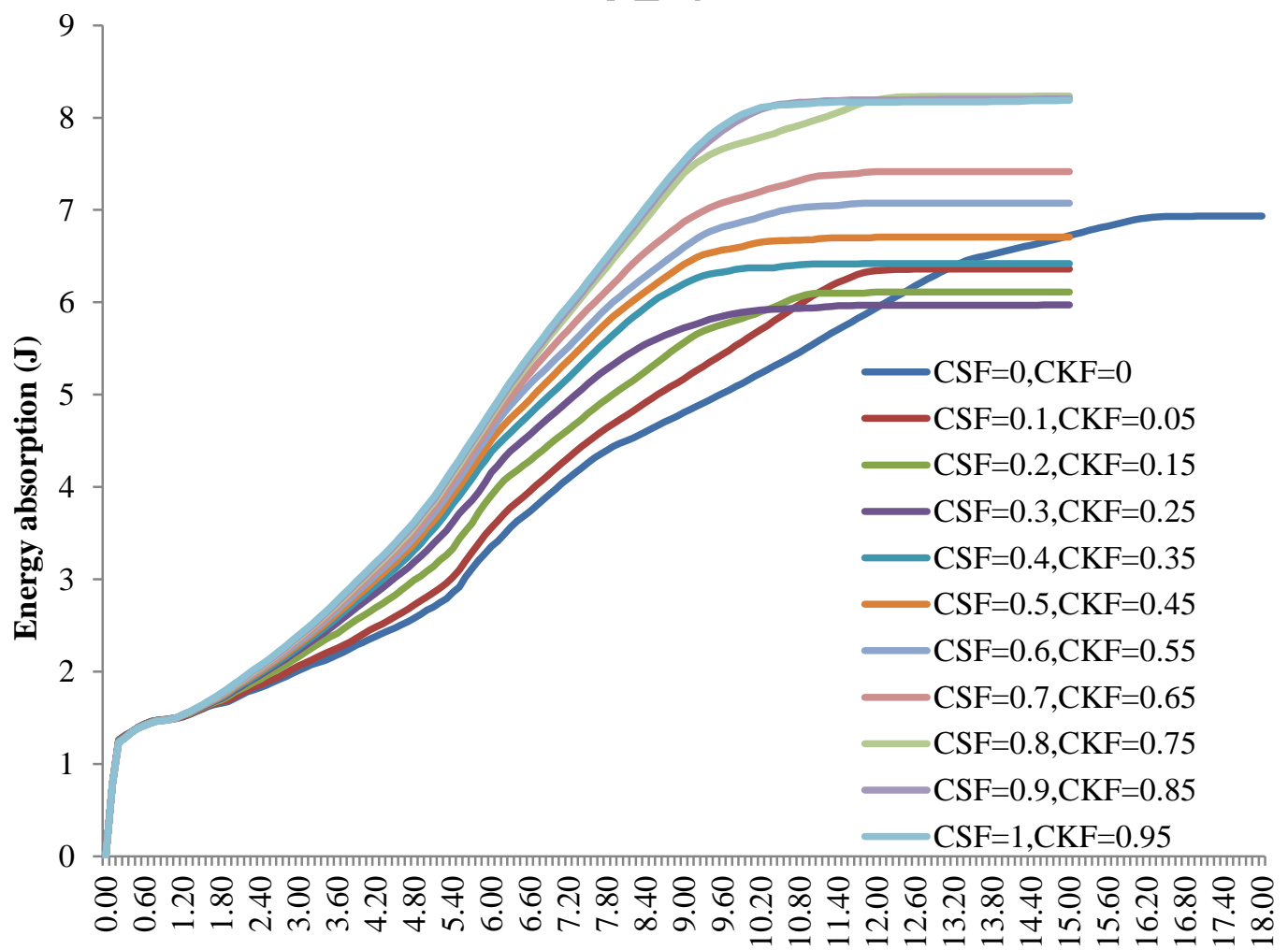

Time ( $\mu$ s)

Figure 14 Time history of energy absorption in TM 72 GPa case 


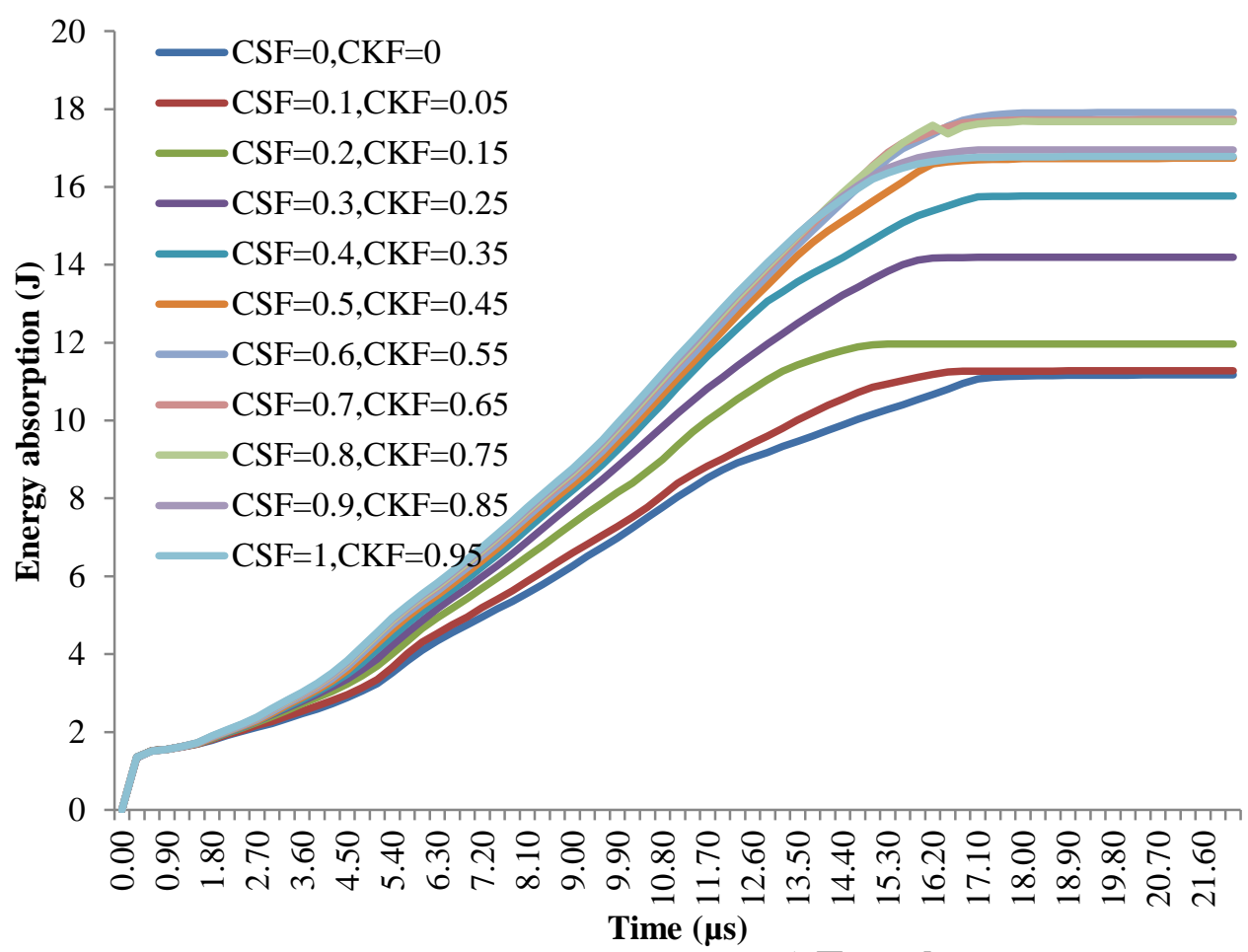

Figure 15 Time history of energy absorption in DYM 112 GPa case

\subsection{Effects of inter-yarn friction on forms of energy absorption}

\subsubsection{The energy transfer between the projectile and the fabric}

The energy dissipation in the fabric takes place mainly in three ways ${ }^{[4-7]}$ : (i) kinetic energy due to the movement of the fabric caused by longitudinal wave and transverse wave; (ii) strain energy due to the deformation of yarns stretched; and (iii) frictional dissipation energy because of inter-yarn friction. When a projectile impacts a fabric, strain is generated in the yarns and part of the energy carried by the projectile is dissipated as strain energy (SE) in the fabric. Meanwhile, the fabric at the impact area would move because of the pulling force of the projectile, leading energy absorbed by the fabric in the form of kinetic energy (KE). Apart from these two routes for dissipation of the projectile energy, inter-yarn friction also plays a role by frictional sliding, which is named as frictional dissipation energy (FDE). 
Figures 16 and 17 describe the percentages of KE, SE and FDE occupied in the overall energy absorption in the two moduli cases. Basically, KE is the dominant energy absorption mechanism and SE and FDE rank the second and third place through the whole process. However, in the friction levels near zero for both models, SE can outperform KE because SE is related to the transverse deflection ability of the fabric and the fabric would deflect most at those extremely low levels of the inter-yarn friction. Thus, the SE at these situations would be the dominant energy absorption form. More energy being absorbed in the form of SE is not desirable because more SE means more transverse deflection of the fabric.

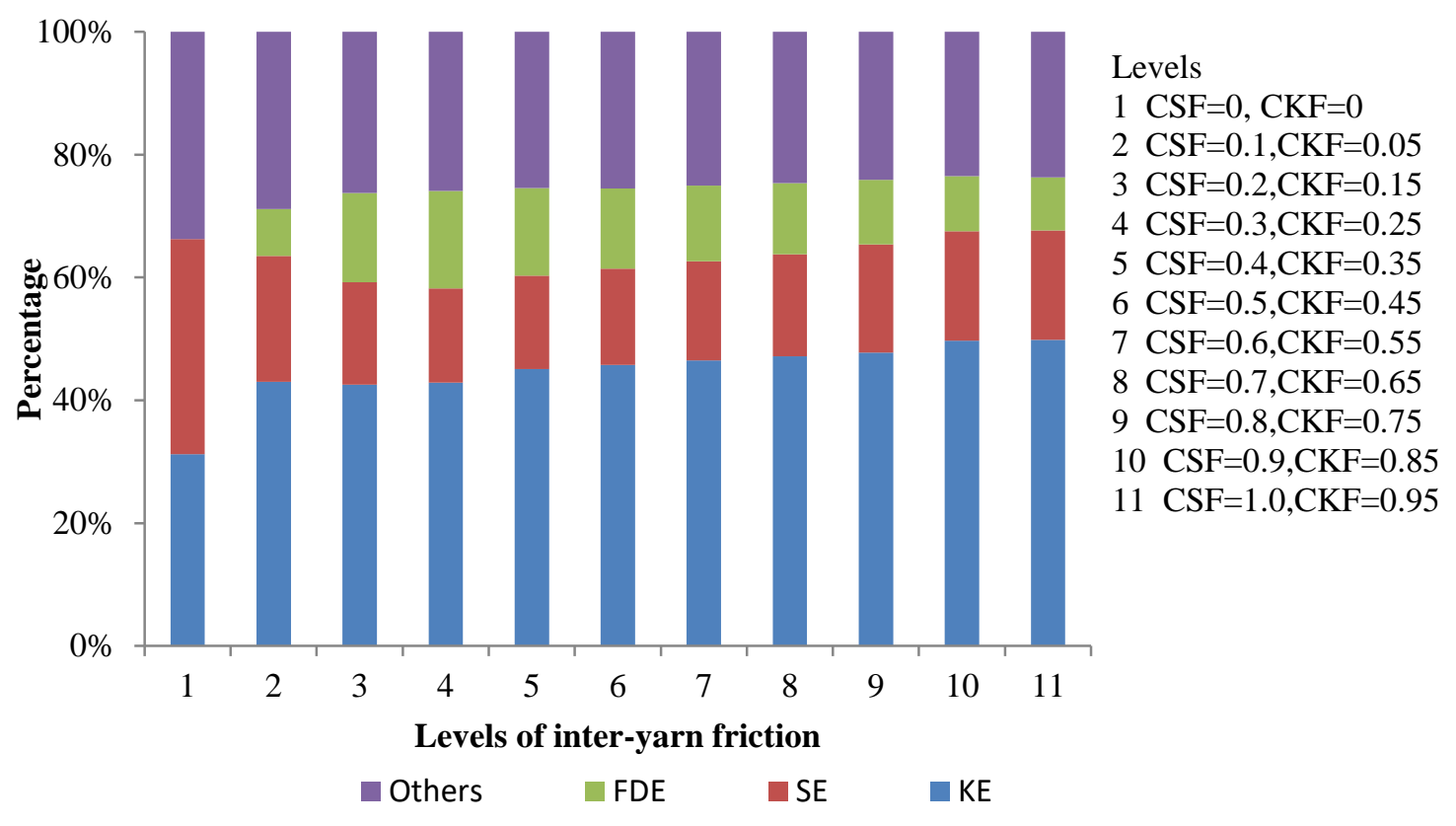

Figure 16 The ultimate percentage of KE, SE and FDE in TM 72GPa case 


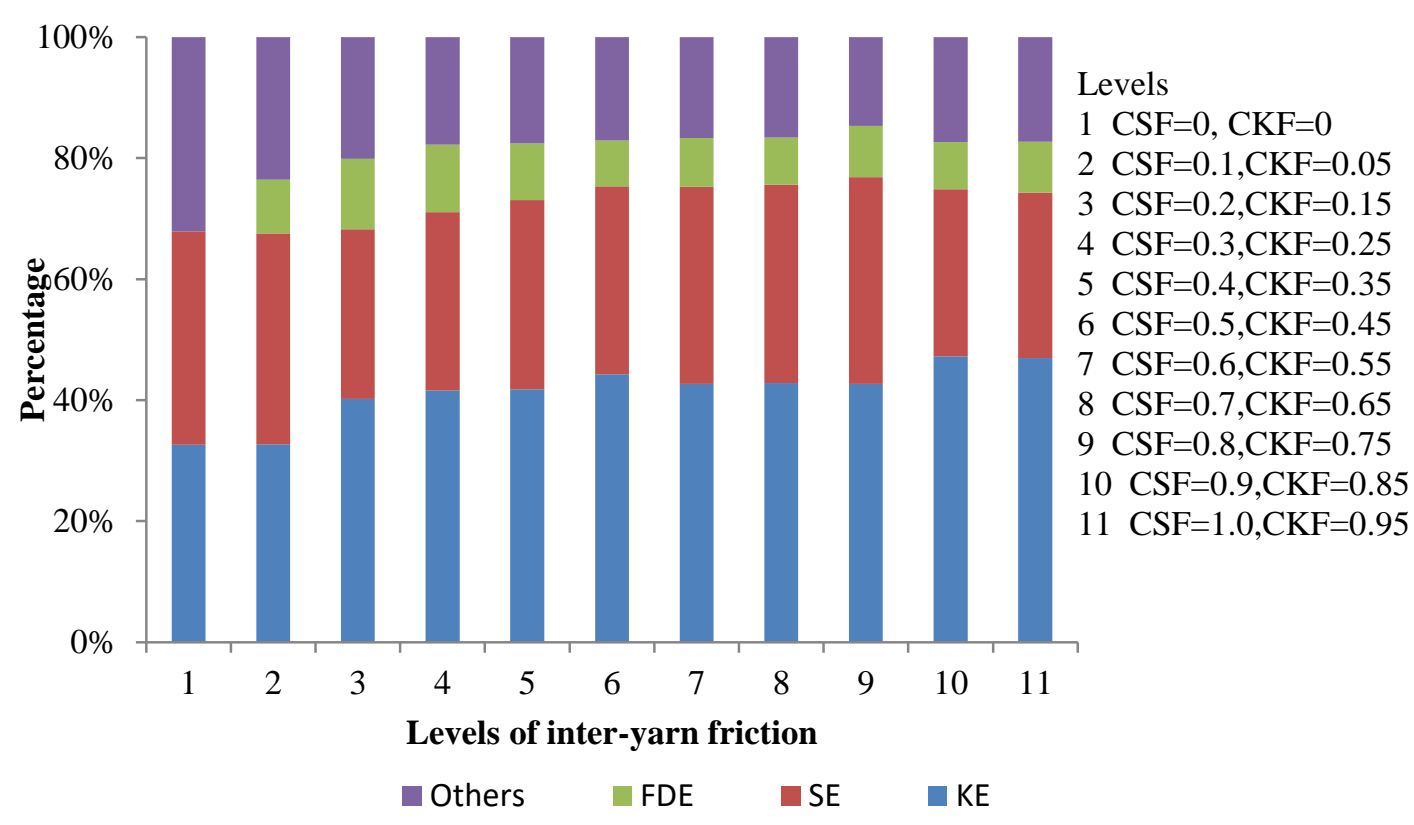

Figure 17 The ultimate percentage of KE, SE and FDE in DYM 112 GPa case

\subsubsection{The kinetic energy}

Figure 18 describes the proportion of KE as function of inter-yarn friction in the two moduli cases. In both cases, the percentages of KE show a slight increasing tendency with the increase of inter-yarn friction. The KE absorption rate also increases with the increase of inter-yarn friction. Higher inter-yarn friction leading to higher $\mathrm{KE}$ absorption rate may be related to the larger transverse wave velocity at higher levels of inter-yarn friction, referring to Figure 8. Larger transverse wave velocity will enable the impact energy to be distributed onto a larger area efficiently. Since KE absorption rate is associated with the involved area at each impact moment, the higher inter-yarn friction would give a higher KE absorption rate, thus higher KE percentage. 


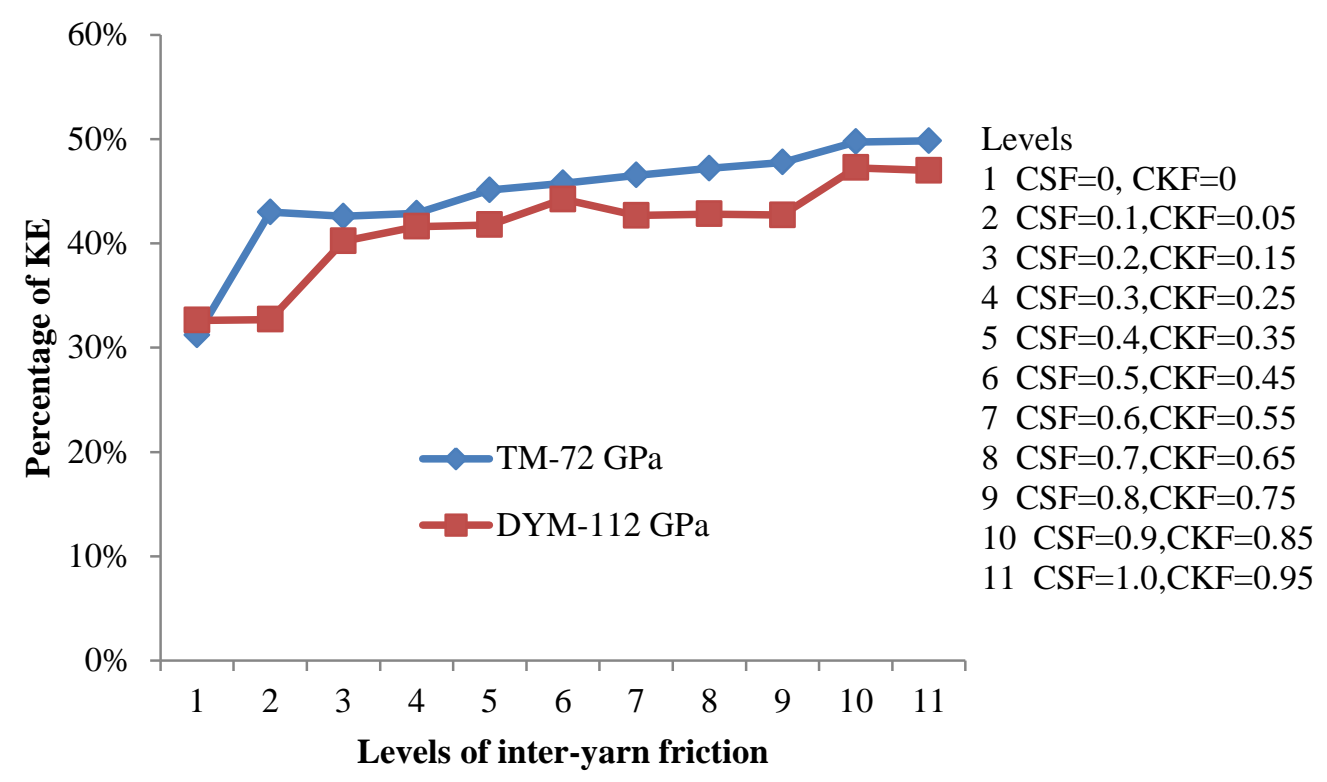

Figure 18The effects of inter-yarn friction on KE

\subsubsection{The strain energy}

Figure 19 shows the effect of inter-yarn friction on the SE percentages in the two moduli cases. The trends of SE percentage as function of inter-yarn friction in two cases are a little different. In DYM case, the amount of SE fluctuates with the increase of inter-yarn friction. The fluctuation is in a small range, around 5\%. Differently, in TM case, the SE percentage dramatically decreases from $35 \%$ to $15 \%$ from zero friction to CSF of 0.30 and CKF of 0.25 . In the rest frictional levels, it is almost kept at that level. It can be concluded that increasing inter-yarn friction to the level of CSF of 0.30 and CKF of 0.25 will significantly decrease SE percentage of the TM fabric than that of the DYM fabric. This may be associated with the difference of transverse deflection ability between these two fabrics. It has been observed that slightly increasing inter-yarn friction will more significantly decreases the transverse deflection ability of TM fabric rather than that of DYM fabric. 


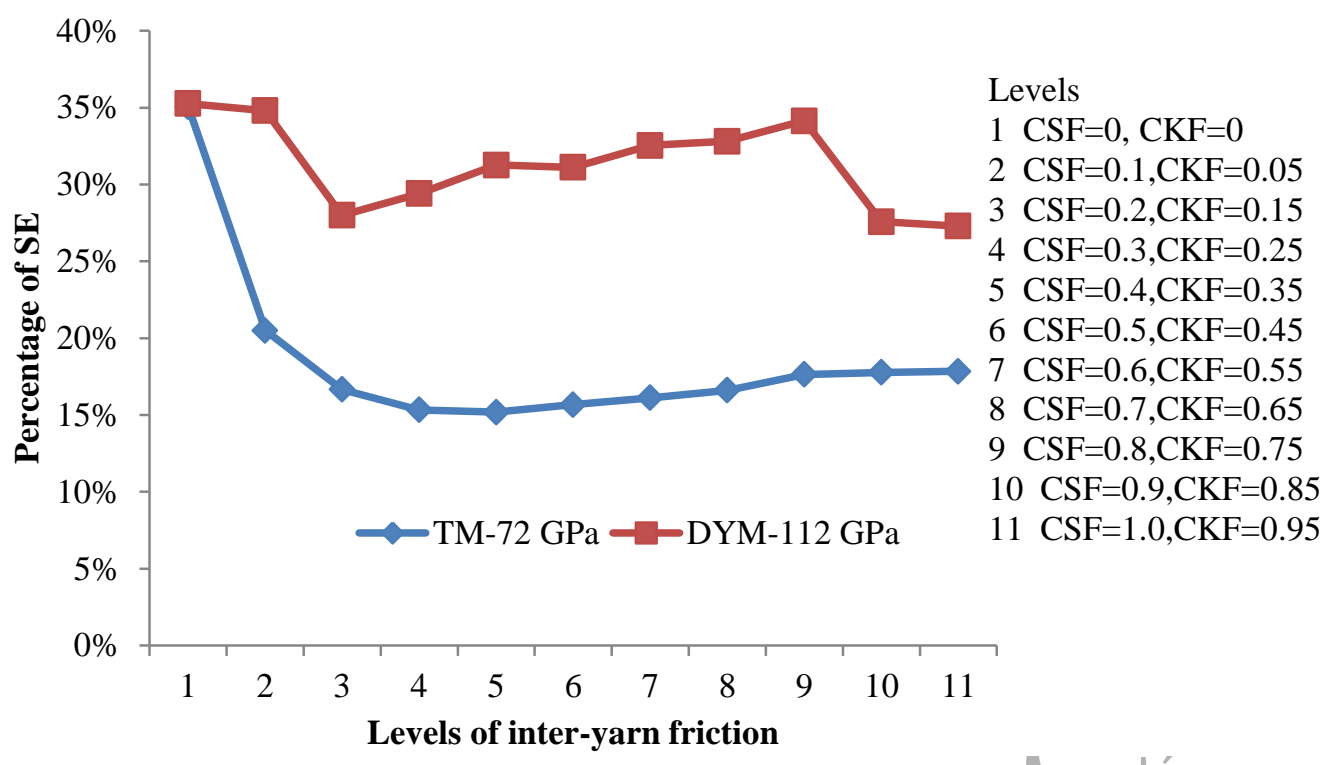

Figure 19 The effects of inter-yarn friction on SE

\subsubsection{The frictional dissipation energy}

The FDE proportion as function of inter-yarn friction is depicted in Figure 20. The proportion of FDE firstly climbs to the peak and then decreases with the increase of inter-yarn friction. To the author's knowledge, the amount of FDE is related to the coefficients of friction as well as the relative motion between yarns. Hence, it is reasonable that with a small increase of inter-yarn friction, the FDE increases dramatically because the work done by friction is linear to the magnitude of frictional force. However, much higher inter-yarn friction would restrain the motions of the yarns, leading to a much lower FDE. An optimum level of inter-yarn friction exists for each fabric to achieve the highest amount of FDE. 


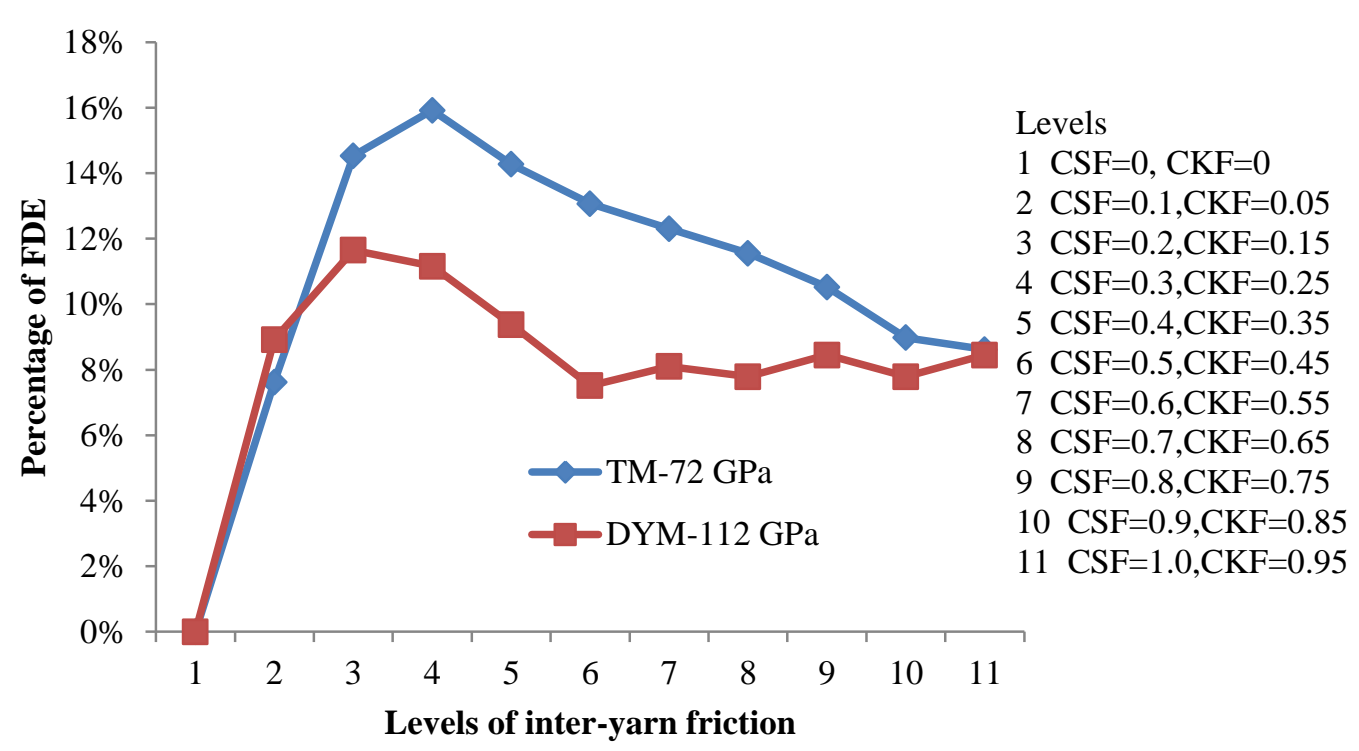

Figure 20 The effects of inter-yarn friction on FDE

\section{Conclusions}

In this chapter, the effects of inter-yarn friction on ballistic performance have been analysed using FE simulation in the two Young's moduli cases of $72 \mathrm{GPa}$ and $112 \mathrm{GPa}$. The effects of inter-yarn friction on the transverse deformation, the overall energy absorption, and the forms of energy absorption during the ballistic impact have been figured out. The main results from the FE theoretical analyses are given below:

(1) Increasing inter-yarn friction decreases the transverse deflection abilities of fabrics.

The response of fabric will transfer from a localised response to a globalised one with the increase of inter-yarn friction, which is more pronounced for the fabric with the yarn of lower Young's modulus.

(2) The transverse deflection ability of the fabric relates to Young's modulus of the yarn. Larger Young's modulus gives rise to lower transverse deflection ability, and vice versa.

(3) The overall energy absorption is associated with the energy absorption rate and the failure time. With the increase of inter-yarn friction, the energy absorption rate is monotonously increased as well while the failure time firstly decreases and then increases but further decreases again.

(4) It is not the desirable energy absorption mode that the TM fabric near zero friction 
absorbs more energy because the mode is a localised response.

(5) Increasing inter-yarn friction can affect the forms of energy absorption. Near zero friction, SE is the dominant mechanism of a fabric. With the increase of inter-yarn friction, KE becomes the dominant one. Moreover, increasing inter-yarn friction can increase KE percentage. Slightly increasing inter-yarn friction will significantly decrease SE percentage of the fabric with yarns of lower Young's modulus but affect little to that of the fabric with yarns of higher Young's modulus. A maximum inter-yarn friction exists for FDE absorption.

Through the theoretical analyses, increasing inter-yarn friction is feasible for improving ballistic performance of body armour constructed by woven fabrics. The practical method for improving ballistic performance of ballistic fabrics can be carried out based on inter-yarn friction mechanism. In this paper, the inter-yarn friction is analysed on the condition of one layer fabric. It is far enough since the ballistic fabrics used for actual ballistic protection is always dozens of layers. In the future, the mechanism of inter-yarn friction for multi-layer fabrics should be investigated.

\section{Acknowledgements}

The authors would like to thank Teijin for proving Twaron ${ }^{\circledR}$ yarns. The Ballistic Research Group at the University of Manchester are also gratefully appreciated. This paper is supported both by Scientific and Technological Guidance project of Chinese Textile Industry Federation (NO. 2017038) and Open Project Program of Provincial Key Laboratory of Functional Textile Material of Henan

\section{References}

[1]Cheeseman BA and Bogetti TA (2003) Ballistic impact into fabric and compliant composite laminates. Composite Structures 61 (1):161-173

[2]Briscoe B and Motamedi F (1992) The ballistic impact characteristics of aramid fabrics: the influence of interface friction. Wear 158 (1):229-247

[3]Hearle J, Leech C, Adeyefa A and Cork C (1981) Ballistic impact resistance of multi-layer textile fabrics. University of Manchester INST of Science and Technology( United Kingdom) DEPT of Textile 
Technology, Manchester

[4]Duan Y, Keefe M, Bogetti T and Cheeseman B (2005) Modeling friction effects on the ballistic impact behavior of a single-ply high-strength fabric. International Journal of Impact Engineering 31 (8):996-1012

[5]Duan Y, Keefe M, Bogetti T, Cheeseman B and Powers B (2006) A numerical investigation of the influence of friction on energy absorption by a high-strength fabric subjected to ballistic impact. International Journal of Impact Engineering 32 (8):1299-1312

[6]Zeng X, Tan V and Shim V (2006) Modelling inter - yarn friction in woven fabric armour. International Journal for Numerical Methods in Engineering 66 (8):1309-1330

[7]Rao M, Duan Y, Keefe M, Powers B and Bogetti T (2009) Modeling the effects of yarn material properties and friction on the ballistic impact of a plain-weave fabric. Composite Structures 89 (4):556-566

[8]Ha-Minh C, Boussu F, Kanit T, Crépin D and Imad A (2012) Effect of frictions on the ballistic performance of a 3D warp interlock fabric: numerical analysis. Applied Composite Materials 19:333-347

[9]Sun D and Chen X (2012) Plasma modification of Kevlar fabrics for ballistic applications. Textile Research Journal 82 (18):1928-1934

[10]Gogineni S, Gao X-L, David N and Zheng J (2012) Ballistic impact of Twaron CT709 ${ }^{\circledR}$ plain weave fabrics. Mechanics of Advanced Materials and Structures 19 (6):441-452

[11]Nilakantan G and Gillespie Jr JW (2012) Ballistic impact modeling of woven fabrics considering yarn strength, friction, projectile impact location, and fabric boundary condition effects. Composite Structures 94 (12):3624-3634

[12]Sun D, Chen X and Mrango M (2013) Investigating ballistic impact on fabric targets with gripping yarns. Fibers and Polymers 14 (7):1184-1189

[13]Sun D, Chen X and Wells G (2014) Engineering and analysis of gripping fabrics for improved ballistic performance. Journal of Composite Materials:1355-1364

[14]Zhou Y, Chen X and Wells G (2014) Influence of yarn gripping on the ballistic performance of woven fabrics from ultra-high molecular weight polyethylene fibre. Composites Part B: Engineering 62:198-204

[15]Wang Y, Chen X, Young R, et al(2016). Finite element analysis of effect of inter-yarn friction on ballistic impact response of woven fabrics[J]. Composite Structures, 135:8-16.

[16]Das S, Jagan S, Shaw A, et al(2015). Determination of inter-yarn friction and its effect on ballistic response of para-aramid woven fabric under low velocity impact[J]. Composite Structures, 120(Available online):129-140.

[17]Tabiei A and Nilakantan G (2008) Ballistic impact of dry woven fabric composites: a review. Applied Mechanics Reviews 61 (1):1-12

[18]Cunniff PM (1992) An analysis of the system effects in woven fabrics under ballistic impact. Textile Research Journal 62 (9):495-509

[19]Chu Y, Min S, Chen X(2017). Numerical study of inter-yarn friction on the failure of fabrics upon ballistic impacts[J]. Materials \& Design, 115:299-316.

[20] Min S , ChuY and Chen X(2016), Numerical study on mechanisms of angle-plied panels for ballistic protection, Materials and Design 90896 - 905

[21]Duan Y, Keefe M, Bogetti T and Powers B (2006) Finite element modeling of transverse impact on a ballistic fabric. International Journal of Mechanical Sciences 48 (1):33-43 
[22]Wang Y, Chen X, Young R, Kinloch I and Wells G (2015) A numerical study of ply orientation on ballistic impact resistance of multi-ply fabric panels. Composites Part B: Engineering 68:259-265 [23]Sun D, Chen X, Lewis E and Wells G (2012) Finite element simulation of projectile perforation through a ballistic fabric. Textile Research Journal 83 (14):1489-1499

[24]Koh ACP, Shim VPW and Tan VBC (2010) Dynamic behaviour of UHMWPE yarns and addressing impedance mismatch effects of specimen clamps. International Journal of Impact Engineering 37 (3):324-332

[25]Huang W, Wang Y and Xia Y (2004) Statistical dynamic tensile strength of UHMWPE-fibers. Polymer 45 (11):3729-3734

[26]Russell B, Karthikeyan K, Deshpande V and Fleck N (2013) The high strain rate response of ultra high molecular-weight polyethylene: from fibre to laminate. International Journal of Impact Engineering 60:1-9

[27]Bouwmeester JGH, Marissen R and Bergsma OK Carbon/dyneeam interlaminar hybrids: new strategy to increae impact resistance or decrease mass of carbon fibre composites. In: 26th international congress of the aeronautical sciences, Anchorage, Alaska, USA, 2008. pp 3851-3856

[28]Lysenko AA, Lysenko VA, Astashkina OV and Gladunova OI (2011) Resource-conserving carbon fibre technologyies. Fibre Chemistry 42 (5):278-286

[29]Technical brochure: Dyneema ${ }^{\circledR}$ in marine and industrial applications. www.dyneema.com. [30]Abaqus Anaylsis User's Manual Volume III Materials. vol III.

[31]Hillerborg A, Modéer M and Petersson P-E (1976) Analysis of crack formation and crack growth in concrete by means of fracture mechanics and finite elements. Cement and concrete research 6 (6):773-781

[32]Talebi H, Wong S and Hamouda A (2009) Finite element evaluation of projectile nose angle effects in ballistic perforation of high strength fabric. Composite Structures 87 (4):314-320

[33]Zee RH and Hsieh CY (1993) Energy loss partitioning during ballistic impact of polymer composites. Polymer Composites 14 (3):265-271

[34]Lee B, Song J and Ward J (1994) Failure of spectra ${ }^{\circledR}$ polyethylene fiber-reinforced composites under ballistic impact loading. Journal of Composite Materials 28 (13):1202-1226

[35]Shim V, Tan V and Tay T (1995) Modelling deformation and damage characteristics of woven fabric under small projectile impact. International Journal of Impact Engineering 16 (4):585-605

[36]Sun C and Potti S (1996) A simple model to predict residual velocities of thick composite laminates subjected to high velocity impact. International Journal of Impact Engineering 18 (3):339-353

[37]Starratt D, Sanders T, Cepuš E, Poursartip A and Vaziri R (2000) An efficient method for continuous measurement of projectile motion in ballistic impact experiments. International Journal of Impact 